\title{
A complete reassessment of standard residual stress uncertainty analyses using neutron diffraction emphasizing the influence of grain size.
}

Robert Charles Wimpory ${ }^{1}$, René V. Martins ${ }^{2}$, Michael Hofmann³, Joana Rebelo Kornmeier ${ }^{3}$, Shanmukha Moturu ${ }^{4}$ and Carsten Ohms ${ }^{5}$

${ }^{1}$ Helmholtz Zentrum Berlin für Materialien und Energie, Hahn-Meitner-Platz 1, 14109 Berlin, Germany

${ }^{2}$ European Commission, Directorate-General for Research \& Innovation, 1049 Brussels, Belgium*

${ }^{3}$ Heinz Maier-Leibnitz Zentrum (MLZ), Technische Universität München, Lichtenbergstr. 1, 85748 Garching, Germany

${ }^{4}$ Advanced Forming Research Centre (AFRC), University of Strathclyde, 85 Inchinnan Drive, Inchinnan, Renfrew PA4 9LJ, United Kingdom

${ }^{5}$ Joint Research Centre, Directorate G - Nuclear Safety and Security, Westerduinweg 3, 1755 LE Petten, The Netherlands

* Disclaimer: "All views expressed herein are entirely of the author, do not reflect the position of the European Institutions or bodies and do not, in any way, engage any of them."

Email addresses:

robert.wimpory@helmholtz-berlin.de, Rene.MARTINS@ec.europa.eu, Michael.Hofmann@frm2.tum.de, joana.kornmeier@frm2.tum.de, Shanmukha.Moturu@strath.ac.uk, Carsten.OHMS@ec.europa.eu

\section{Keywords}

Fitting uncertainty, grain size effect, grain size statistics, slits, spiral slit, oscillating radial collimator, residual strain, residual stress, uncertainty estimation, neutron diffraction, high-energy synchrotron radiation, strain scanning, welding, austenitic steel.

\section{Synopsis}

The peak fitting uncertainty is often not enough to describe completely the true random uncertainty of a neutron strain measurement and resultant stress determinations. Detecting not enough diffracting grains also contributes to the random uncertainty. A simple model to estimate the extra random uncertainty contribution due to the so-called grain size statistics is applied and verified. 


\section{Abstract}

The determination of residual stress in engineering materials with large grains is a challenge when it comes to using diffraction techniques. Not only are the accuracies of the residual stresses themselves important but also the accurate evaluation of their uncertainties. An austenitic steel three-pass slot weld (NeT- TG4) with varying grain size high-lights the potential problems with the data evaluation when grain size is not taken into account whilst measuring strain. Neutron diffraction results are compared with each other (with combinations of slit and radial oscillating collimator beam defining optics) and with high energy synchrotron radiation results with a spiral slit gauge volume defining system. The impact of the grain size on the determination of residual stresses and their associated uncertainties when using diffraction techniques in engineering components is emphasized and discussed. A simple model to estimate the extra random uncertainty contribution due to the so-called grain size statistics is applied and verified. The benefit of continuous or stepwise oscillation to increase the number of detected grains on the detector is discussed together with how to optimize the time of a measurement. From the data obtained, best practice guidelines will be suggested on dealing with large grains when determining strain and stress with neutron diffraction.

\section{Introduction}

In a previous exercise within the first Task Group (TG1) of NeT [1] (The European Network on Neutron Techniques Standardization for Structural Integrity) a single weld bead on plate specimen was measured several times at different facilities. It was noticed that the uncertainty in stress determination quoted, i.e. the one based on standard analysis (using neutron diffraction) tended to be under-estimated [2]: this was especially evident in the weld region and one of the reasons was suspected to be grain size issues as the grains were larger within the weld compared to the parent material.

Subsequently a simple model was developed that indicated the extra uncertainty that was due to grain-size effects [3]. When measuring with a single Bragg peak, the strain in a sample is typically obtained by the fit of a Gaussian function $[4,5,6,7]$ relative to a fit obtained from an appropriate strain free reference sample. The uncertainty of the fit (assuming that the fitting routine is appropriately weighted) is based on counting statistics [8] and this uncertainty propagates through the analyses until the final uncertainty of the stress evaluation [4]. For the counting statistics assumption to be valid there has to be a large enough number of detected diffracting grains within the gauge volume to ensure the representativeness of the observed peak for the entire illuminated gauge volume. The number of detected diffracting grains $N_{D G}$ however, depends on the gauge volume size chosen, the average size and distribution of the grains, and the crystallographic texture within the gauge volume. The simple model, neglecting the crystallographic texture, is hence a function of grain size, gauge volume, Full Width at 
Half Maximum (FWHM) of the Bragg peak, scattering angle $\theta$, size of detector (and hence number of diffracting grains detected) and multiplicity $m^{\text {hkl }}$. It does not take into account the grain shape, i.e. the aspect ratio is taken as 1 . The simple model in [3] does not take into account the mosaicity of the measured sample and is hence implemented here. It is expected that strong preferred orientation (texture) would need an extra factor in the model, which is not included here. The impact of texture however can be minimized by averaging over all measurement directions or measuring a particular reflection with weak texture.

Such a basic model allows the assessment as to whether one can rely on the fitting uncertainty only for a particular sample/material or whether another approach is needed that will increase the effective number of grains seen on the detector, either by oscillating the sample during the measurement or another method. A continuous or stepwise oscillation to increase the number of detected grains on the detector can be used to supress the grain size effect. If the measurement time is too long, the fitting uncertainty (which is time dependent) is no longer representative of the actual uncertainty as the uncertainty due to grain size (which is not time dependent) dominates.

Alternatively the simple model can estimate the extra uncertainty in strain and stress that would be needed in the final stress uncertainty evaluation. One should however be cautious and a suggested upper limit on the largest acceptable uncertainty will be presented.

The work presented here is specific for single peak analysis, for example, when using an angular dispersive neutron diffractometer for the determination of residual stress. The general trends also exist for time of flight (TOF) residual stress diffractometers, but this is beyond the scope of the present work.

Presented here are the results of measurements made on a three pass slot weld, part of the fourth Task Group (TG4) in NeT, which in general involves even larger grain sizes than the single bead on plate predecessor (TG1) [9]. The aim of TG4 within NeT is to undertake 3-dimensional analyses of residual stresses in a three pass slot weld made from austenitic stainless steel $316 \mathrm{~L}$ by both experimental and numerical means. The results show the successful application of the grain-size statistics model on an experimentally challenging engineering material. 


\section{Specimen Description}

The specimens were made from AISI type 316 L austenitic stainless steel. Figure 1 depicts one of the specimens (labelled 3-1A) in-situ in the residual stress neutron diffractometer E3 at the HZB in Berlin, Germany [10]. All specimens were cut from the same steel plate in the same orientation and have dimensions of $18 \mathrm{~mm}$ in thickness, $194 \mathrm{~mm}$ in length and $150 \mathrm{~mm}$ in width. A $6 \mathrm{~mm}$ deep slot is centred on the top face and machined parallel to the plate's longitudinal direction and before welding was about 10 $\mathrm{mm}$ wide and $80 \mathrm{~mm}$ long at the top and $6 \mathrm{~mm}$ wide at its bottom. A semi-automated Tungsten Inert Gas (TIG) welding process was used to deposit the three weld beads on top of each other. Prior to the welding the edges of the plates were machined to obtain rectangular and parallel edges for easier and more reproducible specimen mounting for the strain measurements. More details about the weld can be found in [11]. Figure 2 shows a schematic diagram of the specimens, all specimens being nominally identical. A dedicated protocol was used describing the specimen and the desired measurement points [12]. Each plate has fiducial marks for specimen identification and definition of the planes of interest.

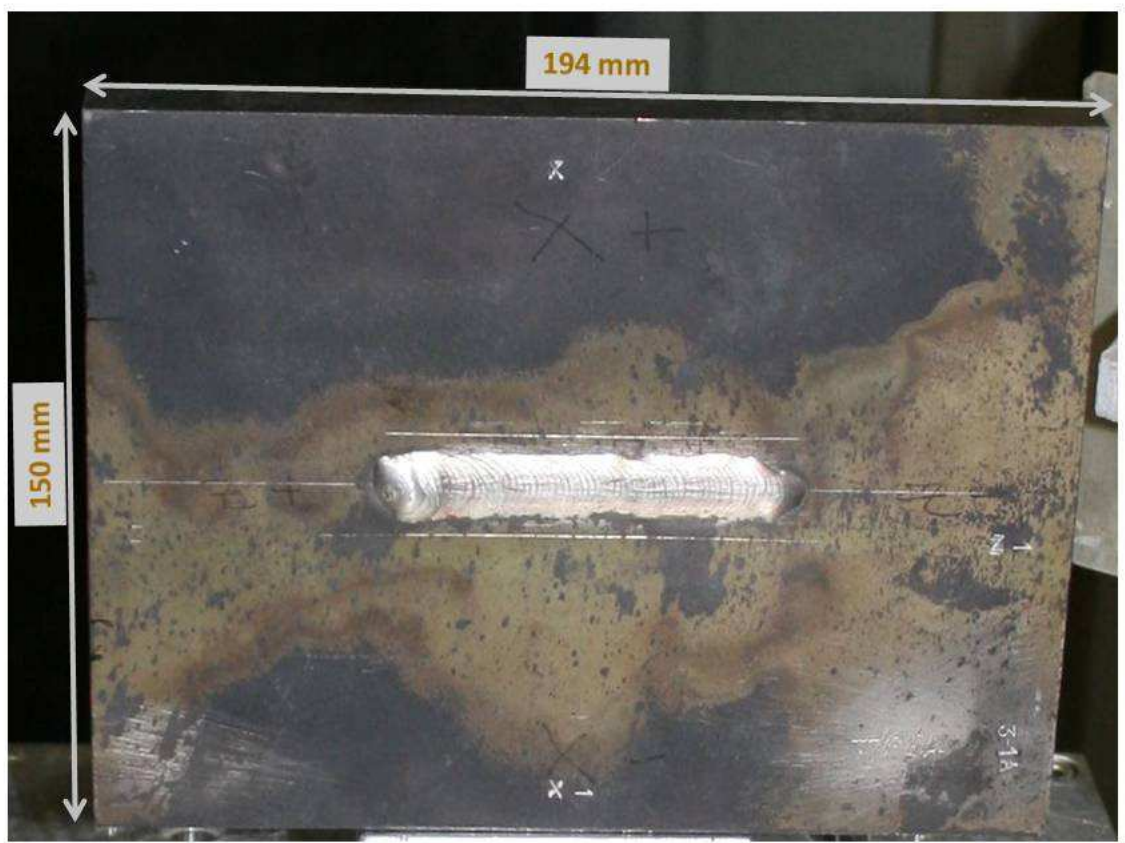

Figure 1. Picture of the TG4 specimen 3-1A in-situ in the residual stress diffractometer $\mathrm{E} 3$ at the HZB, Berlin. 
The location of planes $A, B$, and $C$ (extending in the $x / y$ directions) are at the slot start ( $\mathrm{z}$ $=-40 \mathrm{~mm})$, at the weld mid-length $(z=0 \mathrm{~mm})$, and at the slot end $(z=40 \mathrm{~mm})$ respectively. The plane $D$ extends in directions $y / z$ and contains the longitudinal weld centre line $(x=0 \mathrm{~mm})$. The line $B D$ is defined by the intersection of planes $B$ and $D(x=$ $z=0 \mathrm{~mm}$ ). The origin of the right handed coordinate system is on the top surface in the centre of the plate, y pointing perpendicularly through thickness, $z$ pointing in direction of weld torch traverse, and $x$ perpendicularly to the weld line. Because of the wavy surface of the weld, the origin of the $y$ axis at $x=y=z=0 \mathrm{~mm}$ could not be clearly marked. Therefore, the surface at the centre of the bottom face was defined as position $x=0 \mathrm{~mm}, y=18 \mathrm{~mm}, z=0 \mathrm{~mm}$. Sets of reference coupons, which were cuboids cut using Electric Discharge Machining (EDM) from three regions of one of the specimens accompanied the weld specimen: Parent material (one side coloured black), top $3 \mathrm{~mm}$ of the weld fusion region (one side coloured red) and bottom $3 \mathrm{~mm}$ of the weld fusion region (one side coloured green). Each set of coupons measured $5 \times 8 \times 6 \mathrm{~mm}^{3}$ consisting of four smaller cuboids $\left(5 \times 4 \times 3 \mathrm{~mm}^{3}\right)$ each.

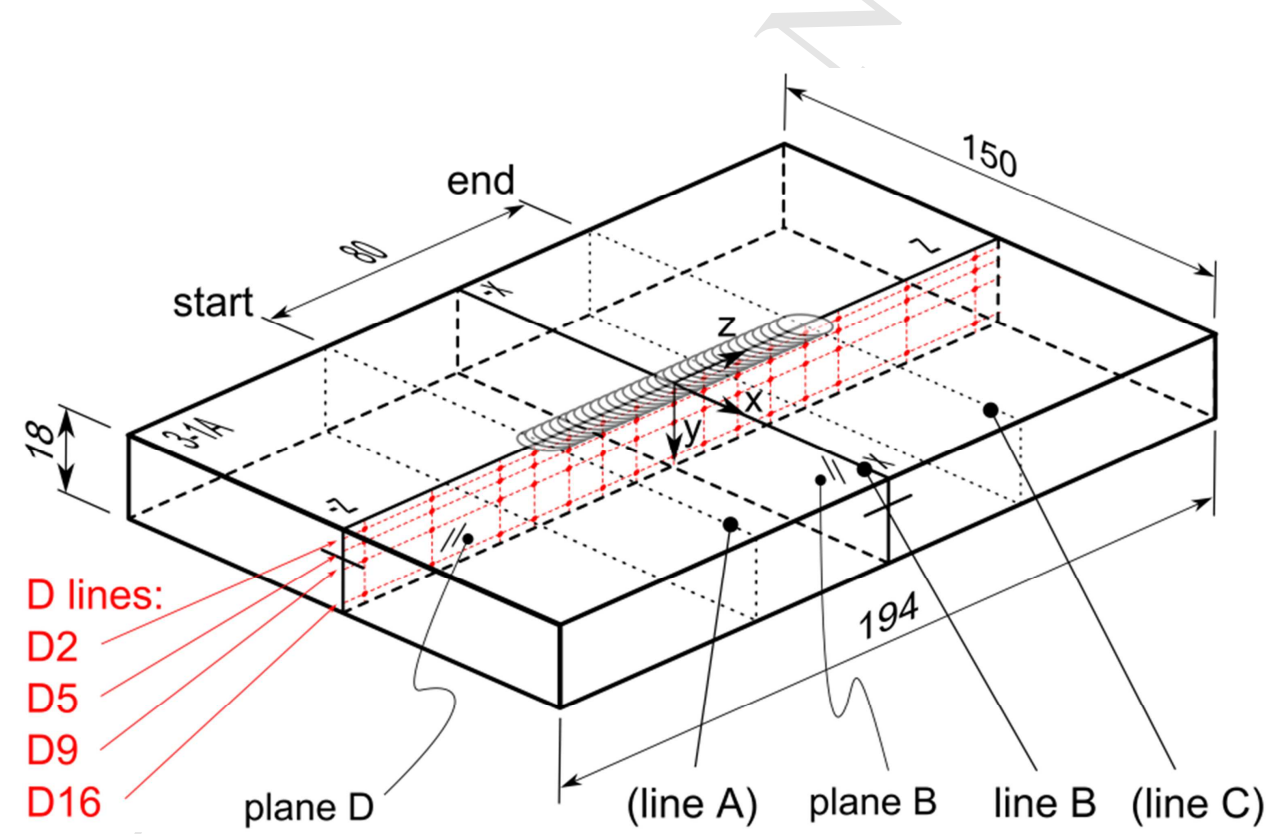

Figure 2. Sketch of TG4 specimen including most of the fiducial marks and the position of specimen identifiers. Start and end refer to the start and end of the slot at $z=-40$ and $+40 \mathrm{~mm}$ respectively. Lines $\mathrm{A}$ and $\mathrm{C}$ mark the intersections of planes $\mathrm{A}$ and $\mathrm{C}$ with the specimen surface at the slot start and end respectively. Line BD is defined as the intersection between planes $\mathrm{B}$ and $\mathrm{D}$. The scan lines depicted, including the neutron diffraction measurement positions $(\bullet)$, are called D2, D5, D9, and D16, according to their $y$-position at 2, 5, 9, and $16 \mathrm{~mm}$ below the top surface. 


\section{Uncertainties in residual stress analysis using diffraction}

\subsection{An overview of conventional uncertainty analyses when using a single Bragg peak}

The neutron diffraction measurements were made on instruments E3 [10] and StressSpec [13] at the steady state neutron sources BER II and FRM II, located at the Helmholtz-Zentrum Berlin and Technische Universität München respectively. In order to measure the strain using neutron diffraction, the specimen is oriented such that the desired measurement direction is aligned with the scattering vector [4], which bisects the incoming and diffracted neutron beams (see Figure 3). The specimen is then positioned such that the centroid of the sampling volume coincides with a chosen measurement location. Subsequently, for this position and orientation the distribution of neutron counts over the angle of scattering is recorded. The duration of neutron counting depends on the required fitting accuracy and the total time available for the measurement campaign. Longer counting leads to better data statistics and results in a lower fitting uncertainty of the data [8].

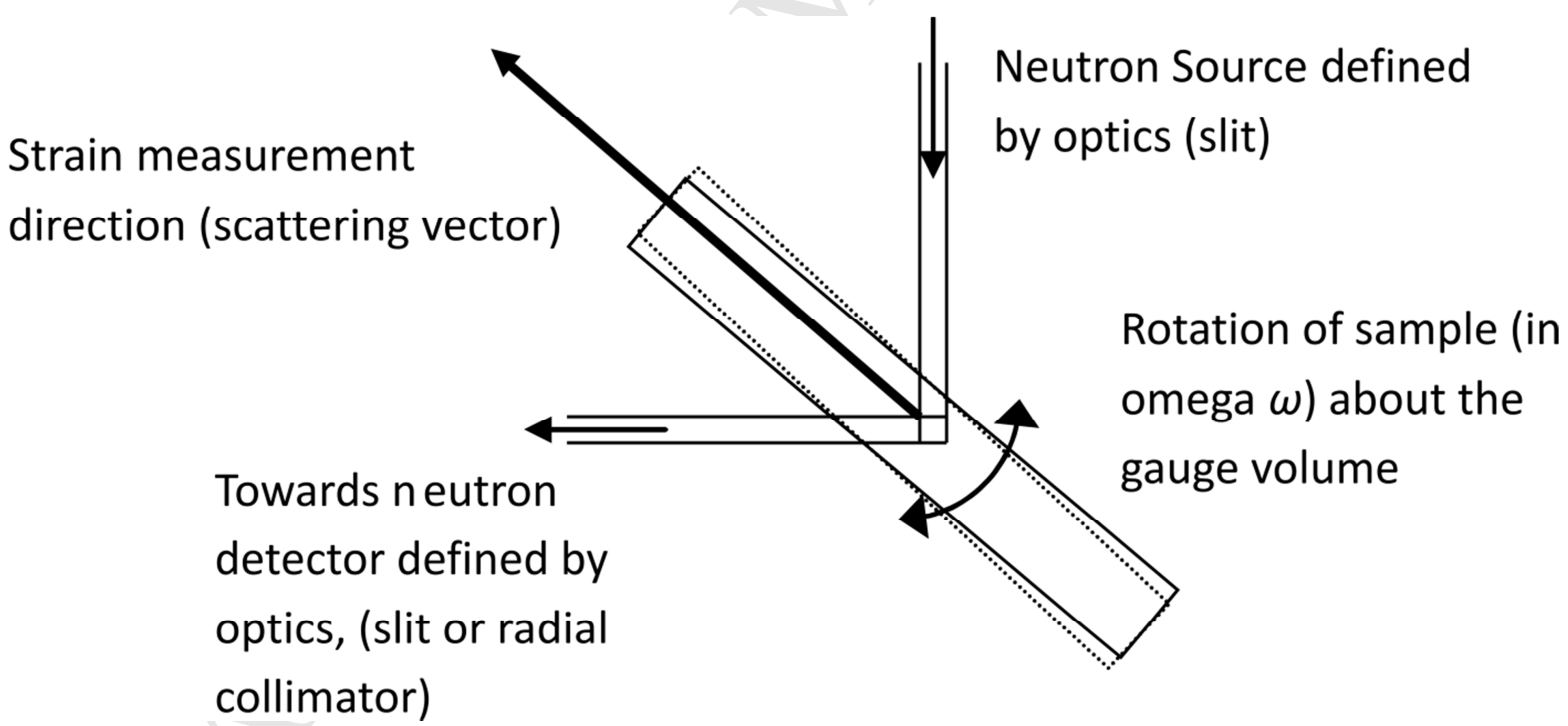

Figure 3. Schematic of a neutron diffraction strain measurement in the weld longitudinal direction. In order to sample more grains, the specimen can be rotated slightly about the omega $(\omega)$ direction (centre of rotation at the centre of the nominal gauge volume), either continuously or stepwise. 
At a steady state reactor the neutrons are typically used in constant wavelength form (monochromatic radiation) and are normally obtained by directing a white neutron beam (containing a range of wavelengths) on a monochromator crystal. The reflected monochromatic beam has a wavelength $\lambda$ and is directed on the specimen where the beam is diffracted. A neutron detector is used to observe the diffracted beam at angle $2 \theta[4,14]$. The observed Bragg peak is normally fitted with a symmetrical Gaussian profile and the change in peak position $\Delta 2 \theta$ with respect to a reference value is used to estimate elastic strain. Thus the strain, $\varepsilon$, (Eq. 1 ) is determined by measuring the $2 \theta$ angle of a material under stress to determine the scattering angle $\theta$, and the $2 \theta_{0}$ angle of the same material not subjected to stress to determine $\theta_{0}$. The uncertainty of the strain is then a combination of the measurement and reference measurement uncertainties, $u\left(2 \theta_{\text {fitting }}\right)$ and $u\left(2 \theta_{0 \text {-fiting }}\right)$ (Eq. 2). These uncertainty values are obtained from the peak fitting routine. The uncertainty in stress is then calculated using Eq. 3. This is based on measuring strain in three orthogonal directions, often the principal strain directions. $E_{h k l}$ and $v_{h k l}$ are the Bragg reflection $\{h k /\}$ specific elastic constants. For the TG4 measurements these values were calculated from single crystal elastic constants for stainless steel 316 [15] based on the self-consistent Eshelby-Kröner model [16]. From now on the subscript ' 0 ' indicates a reference measurement and the absence of subscript indicates a measurement on a sample which is not a reference specimen.

$$
\begin{gathered}
\varepsilon=\frac{\sin \theta_{0}}{\sin \theta}-1 \\
u(\varepsilon)=\frac{1}{\tan \theta_{0}}\left[u\left(\theta_{0-\text { fitting }}\right)^{2}+u\left(\theta_{\text {fitting }}\right)^{2}\right]^{\frac{1}{2}} \\
u\left(\sigma_{x x}\right) \cong\left[\left(\frac{E_{h k l}\left(1-v_{h k l}\right)}{\left(1+v_{h k l}\right)\left(1-2 v_{h k l}\right)} u\left(\varepsilon_{x x}\right)\right)^{2}+\left(\frac{v_{h k l} E_{h k l}}{\left(1+v_{h k l}\right)\left(1-2 v_{h k l}\right)}\right)^{2}\left(u\left(\varepsilon_{y y}\right)^{2}+u\left(\varepsilon_{z z}\right)^{2}\right)\right]^{\frac{1}{2}}
\end{gathered}
$$

$u\left(\theta_{\text {fitting }}\right)$ and $u\left(\theta_{0 \text {-fititing }}\right)$ are half the values of the fitting uncertainties for the $2 \theta$ angle of a material under stress and the same material not subjected to stress, respectively. All angles in the above equations have to be given in radians. Often the same reference value is used throughout a particular region of a sample and can be thus considered a systematic offset (in this case the uncertainty of this offset should be minimized as much as possible). It can be seen from Eq. 2 that the uncertainties of $2 \theta$ angles can be combined in terms of root mean squares (RMS). This is also true for the uncertainties of strains and stresses. 
3.2 Use of a reference specimen to assess the fitting uncertainty accuracy and influence of grain size effect, a reassessment of standard residual stress uncertainty analyses using neutron diffraction.

Measurement of the reference coupons is useful in that it does not only give a strain free reference value but also gives an indication of how the instrument and material behaves while using only little time for the measurement (because of the short neutron path length compared to the corresponding larger specimen). Numerous tests can be carried out simply by rotating and measuring the specimen multiple times, for example in steps of 1 or 2 degrees, as suggested in references [3] and [17].

One can estimate the average number of detected diffracting grains (and hence estimate the grain size in the specimen) and check for possible instrumental aberrations, such as the so called surface effect and its influence if the gauge volume is larger than the specimen. One can also see how well the peak fitting function fits the data and see how representative the fitting routine's estimate of uncertainty is.

One way to test if the fitting uncertainty is correct is to repeat a measurement several times with equal acquisition times, without moving the specimen. The standard deviation of the $2 \theta_{0}$ values should be approximately equal to the average of the fitting uncertainties.

An alternative approach to check if the fitting uncertainty is reasonable is by using Eq. 4 from Withers et. al. [8]. This can expose several possible problems, such as fitting software inaccuracies and whether the actual curve function is appropriate for fitting the Bragg peak (assuming the detector has a linear response and is properly calibrated). The equation is presented for Gaussian peak shapes but it is believed to be approximately correct for a wide range of other common diffraction peak shapes such as Lorentzian, Voigtian etc [8].

$u\left(2 \theta_{\text {fitting }}\right)^{2} \approx\left(\frac{S D_{\text {Gauss }^{2}}}{I}\right)\left[1+2\left(2^{\frac{1}{2}}\right) \frac{B}{H}\right]$

Where $I$ is the integrated area under the peak function (approximately the number of neutrons or photons detected under the peak not including the background). If only the geometrical area under the curve is given by the fit, the integrated intensity is simply the geometrical area divided by the data binning size. $H$ is the height of the peak, i.e. maximum height not including background. $B / H$ is the background/height of peak ratio and $S D_{\text {Gauss }}$ is the peak width and is related to the Full Width at Half Maximum (FWHM) in Eq. 5:

$F W H M=2(2 \ln 2)^{1 / 2} * S D_{\text {Gauss }}$ 
The fitting uncertainty value decreases with an increase of the number of neutrons/photons and thus with time $t$ of measurement. As a rule of thumb, one normally aims to measure $2 \theta$ to an uncertainty of $u(2 \theta) \approx \pm 0.01^{\circ}$ or better (assuming a scattering angle of about $2 \theta \approx 90^{\circ}$ ). The reference value $2 \theta_{0}$ is normally measured to a smaller uncertainty, for instance $u\left(2 \theta_{0}\right) \approx \pm 0.005^{\circ}$.

The total uncertainties $u(2 \theta)$ and $u\left(2 \theta_{0}\right)$ can also contain a significant contribution from so-called grain size statistics [18] as well as from the fitting uncertainty. Normally it is assumed that uncertainties due to sample positioning and instrument calibration are negligible as these should have been thoroughly characterized before the measurement. If all other potential uncertainties are minimized e.g. surface/interface effects, reference value changes (due to composition, micro strain and microstructural variations and possibly texture) then the correct average strain value over which the gauge volume measures within a certain random uncertainty should be achieved. The overall uncertainty in $2 \theta$ and $2 \theta_{0}$ can be described by Eq. $6(a, b)$. Therefore the actual uncertainty in $2 \theta$ and $2 \theta_{0}$ can be thought of comprising two components each. The final strain uncertainty would then take the form of Eq. 7.

$$
\begin{gathered}
u(2 \theta) \approx\left(u\left(2 \theta_{\text {fitting }}\right)^{2}+u\left(2 \theta_{\text {grain }}\right)^{2}\right)^{1 / 2} \\
u\left(2 \theta_{0}\right) \approx\left(u\left(2 \theta_{0-\text { fitting }}\right)^{2}+u\left(2 \theta_{0 \text {-grain }}\right)^{2}\right)^{1 / 2} \\
u(\varepsilon)=\frac{1}{\tan \theta_{0}}\left[u\left(\theta_{\text {fitting }}\right)^{2}+u\left(\theta_{\text {grain }}\right)^{2}+u\left(\theta_{0-\text { fitting }}\right)^{2}+u\left(\theta_{0-\text { grain }}\right)^{2}\right]^{\frac{1}{2}}
\end{gathered}
$$

One way to estimate the actual number of diffracting grains detected on the detector is from Poisson statistics, as demonstrated in [19]. The total integrated intensity I of the neutrons under the Bragg peak has a standard deviation (according to counting statistics) of about $\sqrt{ }(I)$. However, if the actual standard deviation is greater than $\sqrt{ }(I)$ (i.e. the counting statistics assumption is no longer valid) then the concept of Poisson statistics can be used. If one assumes the grains in the gauge volume are Poissondistributed, then one can estimate the actual number of reflecting grains $N_{D G}$ detected on the detector from Eq. 8, where $u(l)$ is the standard deviation of the integrated intensities of multiple measurements and $l$ is the average integrated intensity.

$$
N_{D G} \approx\left(\frac{I}{u(I)}\right)^{2}
$$


The use of Eqs. 6-8 can be demonstrated by using an example of the TG4 black and red reference coupons, representing the parent material and top of weld of the TG4 specimens respectively (Table 1). The black coupon was measured 28 times in steps of 5 degrees in omega $(\omega)$ and the red coupon 109 times in steps of 1.25 degrees in omega $(\omega)$, using a gauge volume of $3 \times 3 \times 2 \mathrm{~mm}^{3}$ using a radial collimator with a fieldof-view FOV $=2 \mathrm{~mm}$ as a secondary optic.

Table 1 shows the different values obtain from the data sets. The value $u\left(2 \theta_{0}\right)$ is the standard deviation of $2 \theta_{0}$ of the multiple measurements, $u\left(2 \theta_{0-\text { fitting }}\right)$ is the expected fitting uncertainty value obtained from Eq. 4 and $u\left(2 \theta_{0 \text {-grain }}\right)$ is the value obtained from Eq. 6(b), the contribution from grain size statistics. In standard analysis one normally assumes $u\left(2 \theta_{0}\right)=u\left(2 \theta_{0 \text {-fiting }}\right)$ but it is apparent that this cannot be assumed when one has a large grain size and efforts have not been made to reduce the grain size contribution. A comparison is made here between the expected value $u\left(2 \theta_{0 \text {-fiting }}\right)$ which is obtained from Eq. 4, independent of the value obtained by the peak fitting routine. The peak fitting software used in this case was StressTextureCalculator [20].

The expected fitting uncertainty derived from Eq. 4 is smaller than that obtained by the fitting program when using a Gaussian peak shape with a linear background. This actually over estimates the fitting uncertainty. Using a pseudo Voigt shape with a linear background in the same program shows a much closer fitting uncertainty compared to the expectation from Eq. 4, suggesting in this case the pseudo Voigt function with a linear background is a more appropriate function to use.

The uncertainties from the fits also have a standard deviation, which have been included in Table 1. This may be a strange concept, a standard deviation of an uncertainty but it shows the spread of values, which increases with the decrease of number of detected diffracted grains $N_{D G}$ (calculated from Eq. 8).

Table 1. Results of TG4 reference specimens (SET Y).

\begin{tabular}{|c|c|c|c|}
\hline TG4 Reference Coupon & Black & Black & Red \\
\hline$\{h k I\}$ & $\{311\}$ & $\{222\}$ & $\{311\}$ \\
\hline Standard deviation of $2 \theta_{0}, u\left(2 \theta_{0}\right)\left[{ }^{\circ}\right]$ & 0.0072 & 0.0116 & 0.0280 \\
\hline Expected fitting value $u\left(2 \theta_{0 \text {-fitting }}\right)\left[^{\circ}\right]$ Eq. 4 & 0.0019 & 0.0049 & 0.0020 \\
\hline $\begin{array}{l}\text { Average fitting uncertainty from software using } \\
\text { Gaussian shape plus linear background }\left[^{\circ}\right]\end{array}$ & $\begin{array}{c}0.0035 \\
\pm 0.0003 \\
\end{array}$ & $\begin{array}{c}0.0058 \\
\pm 0.0013 \\
\end{array}$ & $\begin{array}{c}0.0053 \\
\pm 0.0045 \\
\end{array}$ \\
\hline $\begin{array}{l}\text { Average fitting uncertainty from software using } \\
\text { Pseudo Voigt shape plus linear background }\left[{ }^{\circ}\right]\end{array}$ & $\begin{array}{r}0.0024 \\
\pm 0.0003 \\
\end{array}$ & $\begin{array}{c}0.0051 \\
\pm 0.0010 \\
\end{array}$ & $\begin{array}{r}0.0033 \\
\pm 0.0029 \\
\end{array}$ \\
\hline$u\left(2 \theta_{0 \text {-grain }}\right)\left[{ }^{\circ}\right]$ from Eq. 6. & 0.0069 & 0.0105 & 0.0280 \\
\hline Average Integrated Intensity $I$ of peaks & 13955 & 4928 & 14747 \\
\hline$u(I)$ & 1190 & 884 & 10402 \\
\hline$N_{D G}$ from Eq. 8 & 138 & 31 & 2 \\
\hline
\end{tabular}


It can immediately be seen that the fitting uncertainty is almost negligible compared to that from the grain size contribution for this large grained specimen, even for the parent material with a smaller grain size. Eq. 8 estimates the number of detected diffracting grains $N_{D G}$ and one can see that the $u\left(2 \theta_{0 \text {-grain }}\right)$ value increases as the $N_{D G}$ value decreases. The difference in number of detected diffracting grains is large, being about 138 for the parent material (black reference coupon) and only about 2 for the top of the weld material region (red reference coupon). These are however the $N_{D G}$ values that one would obtain when not oscillating the specimen.

The above measurements are stepwise measurements where the peaks can be added together and fitting the summation of the peaks. Assuming the step size of the omega $(\omega)$ rotation is greater or equal to the mosaicity of the grains, then the black coupon measurement has approximately $28 \times 138=3864$ detected grains and the red coupon $109 \times 2=218$ detected grains after summing the peaks.

This means that the expected fitting uncertainty of the sum of all peaks would be better by a factor of $1 / \sqrt{ }(28)$ and $1 / \sqrt{ }(109)$ for the black and red coupons respectively as the individual peaks were measured with the same time. The fit improves with a factor $1 / N(t)$, where $t$ is time. The uncertainty due to grain size, as shown later, improves with a factor of $\approx 1 / \sqrt{ }\left(N_{D G}\right)$. If we make the same assumption that the omega steps are greater or equal to the mosaicity of the grains, then the uncertainty due to grain size improves by the same factors as for the fitting uncertainty: $1 / \sqrt{ }(28)$ and $1 / \sqrt{ }(109)$ for the black and red coupons respectively. This means for this example the uncertainty due to grain size contribution is still greater than that for the fitting uncertainty in this case.

Oscillating the specimen either stepwise or continuously increases the number of detected grains on the detector. As will be shown later, the time of measurement can be optimised so that the fitting uncertainty is more representative of the total uncertainty.

A simple model based on that described in [3] is shown below. The model in [3] neglected to take into account the mosaicity of the sample. It is now taken into account in the following equation, where $P$ is a probability factor:

$P \approx \frac{\left(D_{H}+\eta_{M}\right) *\left(O S C+\omega_{M}\right)}{4 \pi} * m^{h k l}$

where $D_{H}$ is the angular detector height, $m^{\text {hkl }}$ is the multiplicity of the particular Bragg reflection, $O S C$ is the total angular oscillation of the sample around the $\omega$-axis, $\omega_{M}$ is the 
grain mosaicity around the $\omega$-axis and $\eta_{M}$ is the grain mosaicity in azimuthal direction, i.e. along the diffraction ring. All angular units are converted to radians in Eq. 9.

For a fixed gauge volume, parameter $P$ is proportional to the probability of a grain being seen on the detector. Naturally the larger the detector (and hence $D_{H}$ ), the more grains are detected. When a specimen is not oscillated during a measurement then OSC $=0^{\circ}$, but an oscillation of $\pm 3^{\circ}$ for example (if possible) around the omega $\omega$-axis $\left(O S C=6^{\circ}\right)$, increases the probability of grains being detected. This assumes a $2 \theta$ angle of around $90^{\circ}$. A stepwise oscillation of 5 measurements in $1^{\circ}$ steps when summed is equivalent to $O S C=4^{\circ}$.

The extra random uncertainty due to the grain size can be estimated from Eq. 10 which is a function of $S D_{\text {Gauss, }}$ gauge volume $(g v)$, grain size $\left(S_{G}\right)$ and the probability $P$ from

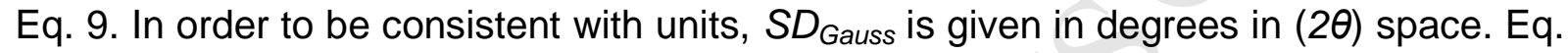
11 shows Eq. 10 solved for grain size. These equations are only approximate and should not be used as an accurate technique to determine grain size. In some cases a grain or grains could be larger than the gauge volume or the gauge volume larger than the specimen being measured. The uncertainty due to grain size $u\left(2 \theta_{\text {grain }}\right)$ is not time dependent and does not improve with time but improves with an increase of the number of detected diffracting grains $\left(N_{D G}\right)$ on the detector.

$$
\begin{aligned}
& u\left(2 \theta_{\text {grain }}\right) \approx \frac{0.5 * S D_{\text {Gauss }}}{\left(N_{D G}\right)^{1 / 2}} \approx \frac{0.5 * S D_{\text {Gauss }}}{\left(P * \frac{g v}{\left(S_{G}\right)^{3}}\right)^{1 / 2}} \\
& S_{G} \approx\left(\frac{P * \text { gv }}{\left(\frac{\left.0.5 * S D_{\text {Gauss }}\right)^{2}}{u\left(2 \theta_{\text {grain }}\right)}\right)^{1 / 3}}\right.
\end{aligned}
$$




\subsection{Calculation of the residual stress uncertainties from synchrotron X-ray diffraction data obtained with the spiral slit technique}

The strain measurements by synchrotron X-ray diffraction were carried out at the highenergy X-ray beamline id15a at the European Synchrotron Radiation Facility (ESRF). The spiral slit technique was employed which is a depth- and phase-resolving nondestructive measurement technique, using focussed monochromatic high-energy $X$ rays, a spiral slit system, and a large area X-ray detector system [11, 21]. Due to the coarse grain structure of the material investigated and the measurement technique used, even with a linear oscillation range of $4 \mathrm{~mm}$ about each measurement position, the diffraction patterns only showed distinct diffraction spots emanating from individual diffracting crystallites. Therefore, "powder like" data analysis approaches were not an option and a novel analysis strategy was applied. As described in detail in [11] this approach was based on the evaluation of the centre of mass position of individual diffraction spots emanating from a specimen volume of interest. Through the geometrical and crystallographic relations every individual diffraction spot position was converted into the lattice parameter ' $a$ ' of the face centred cubic crystal structure of the specimen material. Associated with every a was an uncertainty $u(a)$ which was calculated by propagating the uncertainties of key experimental parameters such as photon wavelength, specimen and detector distances, geometrical errors of the spiral positioning, and errors of the reference $a_{0}$ values through all the equations used for the conversion of the spot position to the lattice parameter a. However, some parameters, such as the spot width, were not included because they are closely linked to the size and orientation of the diffracting crystallite.

The three experimentally accessible in-plane macro-strain tensor components $\varepsilon_{x x}, \varepsilon_{x z}$, and $\varepsilon_{z z}$ (see Figure 2 for definition of coordinate system) of individual gauge volumes were now fitted by solving the over determined system of $M$ linear equations of the form:

$\varepsilon_{x x} f_{x x m}+\varepsilon_{y y} f_{y y m}+\varepsilon_{z z} f_{z z m}+\varepsilon_{x y} f_{x y m}+\varepsilon_{x z} f_{x z m}+\varepsilon_{y z} f_{y z m}=\left(a_{m}-a_{0}\right) / a_{0}$

where $m$ is the index for the grains in the evaluated gauge volume with $m=1$ to $M$, not to be confused with the Bragg peak multiplicity $m^{h k l}$. The $\varepsilon_{i j}$ are the strain tensor components to be fitted and $f_{i j m}$ are specimen and scattering vector orientation depending coefficients. Formulations for the coefficients $f_{i j}$ can be found in the literature e.g.[22]. 
The over determined system of $M$ linear equations was solved by singular value decomposition (SVD) [e.g. 23]. Each individual equation was weighted by the corresponding $u(a)$. The uncertainty $u$ for the resulting strain tensor components was obtained through Eq. 13:

$$
u\left(p_{n}\right)=\sqrt{\sum_{m=1}^{M}\left(\frac{V_{m n}}{w_{n}}\right)^{2}}
$$

where $n$ is the index of the refined parameters with $n=1$ to $N$ (here $N=3$ ), $p_{n}$ is the refined parameter (i.e. $\varepsilon_{i j}$ ), $M$ is the total number of equations contributing to the solution, $w_{n}$ are the singular values as obtained by SVD, and $V_{m n}$ are the elements of the orthogonal matrix V as used in the description of the SVD theorem in Eq. 14:

$$
A=U \cdot W \cdot V^{T}
$$

where $A$ is the $M x N$ design matrix (with $M>M$ ), $U$ the column orthogonal matrix, $W$ the $N \times N$ diagonal matrix with the singular values and $V^{T}$ the transpose of the $N \times N$ orthogonal matrix $V$.

The uncertainties for the stress tensor components were calculated by propagating the uncertainties of the strain tensor components together with a $5 \%$ uncertainty of the Poisson ratio, as prescribed by the measurement protocol [12], through Hooke's law. The final error obtained for each stress tensor component is obviously depending on the number of linear equations used for the determination of the strain tensor components and is scaling with $M^{-1 / 2} . M$ varied between 15 and 1400 , depending on the location in the specimen and the gauge volume size. Note that, as a particularity of the spiral slit technique, the gauge volume dimension parallel to the incoming beam is decided on at the time of the analysis.

An estimation of grain size can also be made and is based on the number of detected diffraction spots, the orientation space data coverage of the recorded data, and the size of the illuminated gauge volume. This is expressed in Eqs. 15 to 20. 


$$
S_{G}=\overline{S_{G}^{h k l}}
$$

Eq. 15 describes that the grain size $S_{G}$ is taken as the average of the grain sizes calculated for specific $\{\mathrm{h} \mathrm{k}\}, S_{G}{ }^{h k l}$. The latter value is calculated following Eq. 16:

$$
S_{G}^{h k l}=\sqrt{\frac{l_{y} \cdot l_{z}}{N_{l G}^{h k l}}}
$$

where $I_{y}$ and $I_{z}$ are the gauge volume dimensions parallel and perpendicular to the beam and $N_{I G}{ }^{h k l}$ is the number of illuminated grains as calculated for a specific $\{\mathrm{h} \mathrm{k} \mathrm{I}\} . I_{z}$ was given by the linear rocking of $4 \mathrm{~mm}$ of the specimen. A dimension in $x$-direction is not taken into consideration because the beam cross section was only $14 \mu \mathrm{m} \times 14 \mu \mathrm{m}$. In fact, the number of grains intersected by the illuminated plane is calculated, rather than the totally illuminated grains in the illuminated volume, which would give erroneous results, due to the small incoming beam dimension. The number of illuminated grains is obtained from Eq. 17:

$$
N_{I G}^{h k l}=\frac{N_{D G}^{h k l}}{m^{h k l} \cdot C_{P F}^{h k l}}
$$

where $N_{D G}{ }^{h k l}$ is the number of detected grains as inferred from the number of detected diffraction spots for a specific $\{\mathrm{h} \mathrm{k} \mid\}, m^{h k l}$ is the multiplicity for the given $\{\mathrm{h} \mathrm{k}\}$ and $C_{P F}{ }^{h k l}$ is the coverage of the $\{\mathrm{h} \mathrm{k}\}$ pole figure by the diffraction data set used.

The coverage is depending on the grain mosaicity and the angular acceptance range of the spiral slit system. This is taken into account in Eq. 18:

$$
C_{P F}^{h k l}=\sin \left(\frac{1}{2} \cdot \omega_{M}^{h k l}\right) \cdot \frac{\left(\eta_{M}^{h k l}+\eta_{S A}\right) \cdot 12}{2 \pi}
$$

where $\omega_{M}$ is the grain mosaicity around the $\omega$-axis (a rotation axis perpendicular to the beam), and is used to determine the slice thickness of the pole figure sphere as filled by one Debye-Scherrer ring. $\eta_{M}$ is the grain mosaicity in azimuthal direction, i.e. along the 
diffraction ring, and $\eta_{S A}$ is the azimuthal acceptance of one spiral, with all angles given in radians. Because the spiral slit system consists of 12 individual slits, a multiplication by 12 is necessary.

$\eta_{S A}$ is given by the geometry of the spiral slit system [11] as described in Eqs. 19 and 20:

$$
\eta_{S A}=\frac{g_{S}}{\sin \left(\arctan \left(\frac{1}{\frac{\pi}{4}+\eta}\right)\right) \cdot r}
$$

$r=r_{0}\left(1+\frac{4}{\pi} \cdot \eta\right)$

where $\eta$ is the azimuthal position on one spiral, $g_{s}$ the spiral slit gap, and $r$ the radial position on the spiral where a specific diffraction signal was accepted by the slit. Eq. 20 is the basic equation of the slit system, describing the form of the spiral, starting at the radius $r_{0}$. For the slit system used the radius and slit gap were $r_{0}=4 \mathrm{~mm}$ and $g_{s}=0.05$ $\mathrm{mm}$. The grain mosaicity in azimuthal direction for grains, within a gauge volume of interest and with a specific $\{\mathrm{h} \mathrm{k}\}$, was estimated by taking the average azimuthal width of all relevant diffraction spots. However, the width used here was not, e.g. the Full Width at Half Maximum, but the width at the peak detection threshold (as used in the first data analysis step consisting of an automatized peak detection procedure), reduced by the constant background, to obtain a better size estimation for the part of the orientation space that is visible through the slit system. Four assumptions were made in calculating the grain sizes in the way described: Firstly, because the specimens were not rotated in this experiment, the grain mosaicity in $\omega$-direction was assumed to be identical to the one in $\eta$-direction. Secondly, it was assumed that no grain gives rise to two diffraction spots simultaneously accepted by the spiral slit system. Thirdly, a random texture was assumed, and lastly, it was assumed that the detection probability is constant for all $\{\mathrm{h} \mathrm{k} \mathrm{l}\}$. The first two assumptions can be considered as being well fulfilled. The last two are more problematic. In fact, the detection probability is not constant, for reasons such as peak intensity detector response and detector noise.

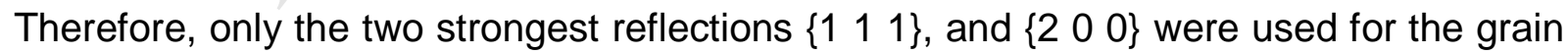
size calculations. The assumption of random texture is well fulfilled in the parent material, as previous texture measurements have shown, but is certainly not fulfilled in the weld region. Stainless steel welds are known to develop a columnar grain structure with $<100>$ growth direction along the maximum heat flow hereby forming a fibre texture [24]. In our specimen this fibre direction is pointing out of the specimen plane, 
almost in the same direction as the incoming $X$-ray beam. This is minimizing the impact of the texture because the scattering vectors are oriented in directions almost at $90^{\circ}$ to the fibre axis. The grain size uncertainties were calculated by propagating the error estimations for $\omega_{M}$ and $\eta_{M}$ through Eqs. 15 to 18 . In our case $\omega_{M}$ and $\eta_{M}$ were identical and they were estimated by taking the standard deviation of the averaged azimuthal widths for a specific $\{\mathrm{h} \mathrm{k} \mid\}$ and position.

\section{Measurement protocol}

For the high energy synchrotron radiation measurements a complete mapping of the specimen 3-1A was performed in the transverse and longitudinal strain directions, including all the measurements points stipulated in the measurement protocol [12]. For the stress evaluation, the normal stress component was assumed to be zero.

For the neutron measurements a more limited set of measurements points were measured. In each case three orthogonal strain directions were measured to determine the stress. Only the data from three $D$ lines are compared to the high energy synchrotron data.

Table 2 shows some of the experimental parameters used for the D-line strain measurements. Following the protocol [12] the $D$ lines were measured and four sets of data from the different instruments used different measuring parameters. The $D$ lines that are compared here are D2 with $x=0, y=2$, D5 with $x=0, y=5$ and D9 with $x=0$, $y=9$, each with strain measurements and stress determinations at positions $z=-90,-70,-50,-45,-40,-35,-30,-20,-10,0,1020,30,35,40,45,50,70$ and 90 $\mathrm{mm}$. Figure 2 shows the location of these points. All measurements presented were made on specimen labelled 3-1A.

The first Stress-Spec measurements (labelled FRM II (a)) used the smallest gauge volume and the smallest oscillation and so any potential grain-size issues should be the most apparent. The HZB E3 measurements use the largest gauge volume and the largest oscillation and so the grain-size issues should be the least. The measurement made at the HZB was continuously oscillated $+/-5^{\circ}$ in omega ( $\omega$-axis), hence $O S C=10^{\circ}$, during data acquisition using slit optics of $3 \times 3 \times 3 \mathrm{~mm}^{3}$. This meant that more grains are observed on the detector. The second Stress-Spec measurement (labelled FRM II (b)), used a radial oscillating collimator as a secondary optic instead of a slit [25]. Of the three existing reference specimen batches produced the one measured by HZB carried the identification mark $W$, and the one measured at the FRM II and at the ESRF experiments, the mark $Z$. 
Several different measurements were also made on the three reference specimens (TG4-SET Y) on E3 in a stepwise manner around the $\omega$-axis. The reference coupons are cuboids cut using Electric Discharge Machining (EDM) from three regions of the specimen (Parent material, top $3 \mathrm{~mm}$ of the weld fusion region and bottom $3 \mathrm{~mm}$ of the weld fusion region). Each set of coupons measured $5 \times 8 \times 6 \mathrm{~mm}^{3}$ consisting of four smaller cuboids $\left(5 \times 4 \times 3 \mathrm{~mm}^{3}\right)$ each. The coupons were orientated so that the $8 \mathrm{~mm}$ side pointed up.

Table 2. Comparisons of measurement set-ups for the D-lines on the 3-1A Specimen

\begin{tabular}{|c|c|c|c|c|}
\hline $\begin{array}{l}\text { Laboratory } \\
\text { (Instrument) }\end{array}$ & $\begin{array}{l}\text { Optics } \\
{\left[\mathrm{mm}^{3}\right]}\end{array}$ & $\begin{array}{c}\text { Omega }(\omega) \\
\text { Oscillation }\left[{ }^{\circ}\right]\end{array}$ & No. of peaks & 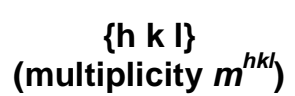 \\
\hline $\begin{array}{c}\text { FRM II (a) } \\
\text { (Stress-Spec) }\end{array}$ & $\begin{array}{l}2 \times 2 \times 2 \text { (input and } \\
\text { output slits) }\end{array}$ & $\begin{array}{l}\text { Yes. } \pm 3 \\
\text { OSC }=6\end{array}$ & 1 & $\left\{\begin{array}{lll}3 & 1 & 1\end{array}\right\}(24)$ \\
\hline $\begin{array}{c}\text { FRM II (b) } \\
\text { (Stress-Spec) }\end{array}$ & $\begin{array}{c}3 \times 3 \times 2.1 \text { (input slit } \\
\text { and Radial oscillating } \\
\text { on output) }\end{array}$ & $\begin{array}{l}\text { Yes. } \pm 4 \\
\text { OSC }=8\end{array}$ & 1 & $\left\{\begin{array}{lll}3 & 1 & 1\end{array}\right\}(24)$ \\
\hline $\begin{array}{l}\text { HZB } \\
\text { (E3) }\end{array}$ & $\begin{array}{c}3 \times 3 \times 3 \text { (input and } \\
\text { output slits) }\end{array}$ & $\begin{array}{l}\text { Yes. } \pm 5 \\
O S C=10\end{array}$ & 1 & $\left\{\begin{array}{lll}3 & 1 & 1\end{array}\right\}(24)$ \\
\hline $\begin{array}{c}\text { JRC at ESRF made on } \\
\text { ID15a } \\
\text { High Energy } \\
\text { Synchrotron X-rays }\end{array}$ & $\begin{array}{l}\text { Special spiral slit set- } \\
\text { up }\end{array}$ & No & 5 & $\begin{array}{l}\left\{\begin{array}{llll}1 & 1 & 1\end{array}\right\}(8) \\
\left\{\begin{array}{lll}2 & 0 & 0\end{array}(6)\right. \\
\left\{\begin{array}{lll}2 & 2 & 0\end{array}\right\}(12) \\
\left\{\begin{array}{llll}3 & 1 & 1\end{array}\right\}(24) \\
\left\{\begin{array}{lll}2 & 2 & 2\end{array}\right\}\end{array}$ \\
\hline
\end{tabular}

\section{Results and Discussion}

\subsection{Reference specimens TG4 SET Y, Optimizing the time of measurement}

The TG4 reference sample (parent material SET Y) was placed upright with a gauge volume of $2 \times 2 \times 2 \mathrm{~mm}^{3}$ on E3 at the HZB, Berlin. The sample was measured in steps of $1^{\circ}$ from $-155^{\circ}$ to $+63^{\circ}$ about the sample $\omega$-axis resulting in 219 measurements. The $\{3$ $11\}$ and $\left\{\begin{array}{l}22 \\ 2\end{array}\right\}$ reflections were measured simultaneously and the corresponding data are presented in Tables 3 and 4 respectively. The reflections have different multiplicities, $m^{311}=24$ and $m^{222}=8$. Each single measurement took an average of 382 seconds. The $\left\{\begin{array}{lll}3 & 1 & 1\end{array}\right\}$ rate of improvement of fitting uncertainty is relatively fast (see Figure 4.) because of the short path length of the neutrons in the small sample. A different curve would exist for different materials, neutron path lengths, detector sizes etc. 
As seen in section 3, for a particular measurement point the rate at which the fitting uncertainty improves (decreases) depends on the neutron flux on the sample, absorption of neutrons in the sample (path length of the neutrons), scattering properties of the material, gauge volume, FWHM, texture, multiplicity $\left(m^{h k l}\right)$, detector size and signal to noise ratio $\mathrm{B} / \mathrm{H}$. For a particular measurement point only the integrated area $I$ changes over time, thus (assuming the neutron source is stable) as $I$ is proportional to time $t$ the fitting uncertainty improves with a factor $1 / \sqrt{ }(t)$.

The first row in Table 3 shows the difference between the standard deviation of the $2 \theta_{0}$ values of the 219 peaks compared to the expected fit value (after 382 seconds). An estimation of the $N_{D G}$ value was calculated using Eq. 8. For instance, for the 219 single peaks using the $\left\{\begin{array}{lll}3 & 1 & 1\end{array}\right\}$ reflection, the average value of $I$ is 6751 and the standard deviation is $u(I)=684$. Using Eq. 8 results in a value of $N_{D G}=97$. Summing the peaks in ' $2 s$ ' and ' $3 s$ ' etc. (rows 2 and 3 in Table 3), increases the value of $N_{D G}$, this is equivalent to oscillating the specimen. Summing in this way with these particular measurement time intervals and experimental set-up show that the actual grain size uncertainty is always about threes time more than the fitting uncertainty which means the measurement time used was far too high.

Summing the peaks in twos (row 2 in Table 3 ), for example, is equivalent to a continuous oscillation of $\pm 0.5^{\circ}(\mathrm{OSC}=1)$ as the steps used were $1^{\circ}$, below the expected mosaicity of the material which is estimated to be about $1.2^{\circ}$. As a typical value of mosaicity a default value of $\omega_{M}=\eta_{M}=1.2^{\circ}$ was used. Measurements made at the ESRF by the JRC saw a range of mosaicity values of up to $1.55^{\circ}$ for the $\left\{\begin{array}{lll}1 & 1 & 1\end{array}\right\}$ reflection.

One can use the results to optimise the measurement time so that the grain size contribution is no longer dominant. Figure 4 shows that for this particular experimental set-up, one only needs about 40 seconds to get below a value fitting uncertainty of $u\left(2 \theta_{\text {o-fiting }}\right) \approx \pm 0.01^{\circ}$. If one does not oscillate then one has a value $N_{D G} \approx 97$. The total uncertainty cannot get lower than about $\pm 0.0115^{\circ}$ no matter how long one measures for because of the $u\left(2 \theta_{0 \text {-grain }}\right)$ contribution. In this case one should not measure longer than 40 seconds otherwise the value of $u\left(2 \theta_{0 \text {-grain }}\right)$ will dominate.

Oscillation of the specimen with this particular set-up (either continuously $\mathrm{OSC}=3$ or stepwise of 4 measurements in $1^{\circ}$ steps, see row 4 in Table 3) results with $N_{D G} \approx 284$ and the total uncertainty cannot get lower than $\pm 0.0049^{\circ}$ after about 200 seconds. Therefore one should not measure longer than 200 seconds otherwise the value of $u\left(2 \theta_{0 \text {-grain }}\right)$ will dominate. 
One can estimate the size of the grains $S_{G}$ from Eq. 11, this is just to cross check the model. One should get a similar value for each summation, which indeed one does get. A value of $S_{G}$ of around $87-100 \mu \mathrm{m}$ is the estimate for the parent material.

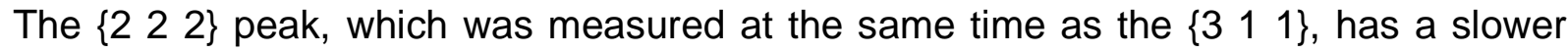
fitting uncertainty improvement rate and also the specimen needs to be oscillated much more to achieve a big enough value of $N_{D G}$.

The multiplicity of $\{222\}$ is 8 and so 3 times less grains should be detected (assuming no texture) compared to the $\left\{\begin{array}{lll}3 & 1 & 1\end{array}\right\}$. It appears that in this case there is texture, as about 5 times less grains are detected.

Table 4 shows that not oscillating the specimen $(\mathrm{OSC}=0)$ results in a value of only $N_{D G}$ $\approx 23$ which means one cannot measure better than a value of $u\left(2 \theta_{0}\right) \approx \pm 0.0235^{\circ}$. In this case it is not worth measuring much longer than 30 seconds; however $\pm 0.0235^{\circ}$ is a rather poor uncertainty. Oscillation of the specimen (either continuously $\mathrm{OSC}=3$ or stepwise of 4 measurements in $1^{\circ}$ steps, see row 4 in Table 4) results with $N_{D G} \approx 54$ and the total uncertainty cannot get lower than $\pm 0.0124^{\circ}$ after about 100 seconds. Therefore one should not measure longer than 100 seconds otherwise the value of $u\left(2 \theta_{0 \text {-grain }}\right)$ will dominate.

Measurement of the larger TG4 weld specimen under the same conditions could also be optimised in a similar fashion, where the rate of improvement of fitting uncertainty will be slower (due to increased neutron path length) but the uncertainties due to grain size are the same.

Table 3. Stepwise oscillation measurements of the black parent reference coupon using the $\left\{\begin{array}{lll}3 & 1 & 1\end{array}\right\}$ reflection. Average FWHM of peaks $=0.52^{\circ} . N_{D G}$ is estimated from Eq. 8 , $u\left(2 \theta_{0}\right)$ is the standard deviation of the $2 \theta_{0}$ values, $u\left(2 \theta_{0 \text {-fiting }}\right)$ is estimated from Eq.4, $u\left(2 \theta_{0 \text {-grain }}\right)(a)$ is from Eq. $6(\mathrm{~b})$ and $u\left(2 \theta_{0 \text {-grain }}\right)(b)$ is estimated from the model Eq. $10 . P$ is determined using Eq. 9, and $S_{G}$ is determined using Eq. 11.

\begin{tabular}{|c|c|c|c|c|c|c|c|c|c|c|}
\hline row & $\mathbf{N}_{\mathrm{DG}}$ & $\begin{array}{l}\text { No. of } \\
\text { peaks }\end{array}$ & $\begin{array}{c}\text { OSC } \\
\left.{ }^{\circ}\right]\end{array}$ & $\begin{array}{c}\text { Average } \\
2 \theta_{0} \\
{\left[{ }^{\circ}\right]}\end{array}$ & $\begin{array}{c}\mathrm{u}\left(2 \theta_{0}\right) \\
{\left[{ }^{\circ}\right]}\end{array}$ & $\begin{array}{c}u\left(2 \theta_{0 \text {-fitting }}\right) \\
{\left[^{\circ}\right]}\end{array}$ & $\begin{array}{c}u\left(2 \theta_{0-\text { grain }}\right) \\
(\text { a) } \\
{\left[{ }^{\circ}\right]}\end{array}$ & $\begin{array}{c}\mathrm{u}\left(2 \theta_{0 \text {-grain }}\right) \\
(\mathrm{b}) \\
{\left[^{\circ}\right]}\end{array}$ & $\mathbf{P}$ & $\begin{array}{c}\mathrm{S}_{\mathrm{G}} \\
{[\mu \mathrm{m}]}\end{array}$ \\
\hline 1 & 97 & 219 & 0 & 85.3688 & 0.0120 & 0.0037 & 0.0115 & 0.0111 & 0.0115 & 100 \\
\hline 2 & 183 & 109 & 1 & 85.3688 & 0.0077 & 0.0026 & 0.0073 & 0.0081 & 0.0210 & 91 \\
\hline 3 & 241 & 73 & 2 & 85.3688 & 0.0065 & 0.0021 & 0.0061 & 0.0070 & 0.0306 & 92 \\
\hline 4 & 284 & 54 & 3 & 85.3687 & 0.0053 & 0.0018 & 0.0049 & 0.0065 & 0.0401 & 87 \\
\hline 5 & 297 & 43 & 4 & 85.3687 & 0.0048 & 0.0016 & 0.0045 & 0.0063 & 0.0497 & 87 \\
\hline
\end{tabular}




\begin{tabular}{c|c|c|c|c|c|c|c|c|c|c}
\hline $\mathbf{6}$ & 350 & 36 & 5 & 85.3687 & 0.0050 & 0.0015 & 0.0048 & 0.0058 & 0.0592 & 97 \\
\hline $\mathbf{7}$ & 373 & 31 & 6 & 85.3687 & 0.0045 & 0.0014 & 0.0043 & 0.0057 & 0.0687 & 95 \\
\hline $\mathbf{1 0}$ & 432 & 21 & 9 & 85.3688 & 0.0035 & 0.0011 & 0.0033 & 0.0053 & 0.0974 & 89
\end{tabular}

Table 4. Stepwise oscillation measurements of the black parent reference coupon using the $\left\{\begin{array}{lll}2 & 2 & 2\end{array}\right\}$ reflection. Average FWHM of peaks $=0.57^{\circ} N_{D G}$ is estimated from Eq. 8, $u\left(2 \theta_{0}\right)$ is the standard deviation of the $2 \theta_{0}$ values, $u\left(2 \theta_{0 \text {-fiting }}\right)$ is estimated from Eq.4, $u\left(2 \theta_{0 \text {-grain }}\right)(a)$ is from Eq. $6(\mathrm{~b})$ and $u\left(2 \theta_{0 \text {-grain }}\right)(b)$ is estimated from the model Eq. $10 . P$ is determined using Eq. 9, and $S_{G}$ is determined using Eq. 11.

\begin{tabular}{|c|c|c|c|c|c|c|c|c|c|c|}
\hline row & $N_{D G}$ & $\begin{array}{l}\text { No. of } \\
\text { peaks }\end{array}$ & $\begin{array}{c}\text { OSC } \\
{\left[^{\circ}\right]}\end{array}$ & $\begin{array}{c}\text { Average } \\
2 \theta_{0} \\
{\left[{ }^{\circ}\right]}\end{array}$ & $\begin{array}{c}u\left(2 \theta_{0}\right) \\
{\left[{ }^{\circ}\right]}\end{array}$ & $\begin{array}{c}u\left(2 \theta_{0 \text {-fitting }}\right) \\
{\left[^{\circ}\right]}\end{array}$ & $\begin{array}{c}\mathbf{u}\left(2 \theta_{0 \text {-grain }}\right) \\
(\mathrm{a}) \\
{\left[{ }^{\circ}\right]}\end{array}$ & $\begin{array}{c}\mathbf{u}\left(2 \theta_{0 \text {-grain }}\right) \\
\text { (b) } \\
{\left[^{\circ}\right]}\end{array}$ & $\mathbf{P}$ & $\begin{array}{c}S_{G} \\
{[\mu \mathrm{m}]}\end{array}$ \\
\hline 1 & 23 & 219 & 0 & 90.1512 & 0.0260 & 0.0113 & 0.0235 & 0.0249 & 0.0038 & 106 \\
\hline 2 & 38 & 109 & 1 & 90.1512 & 0.0176 & 0.0080 & 0.0157 & 0.0194 & 0.0070 & 99 \\
\hline 3 & 49 & 73 & 2 & 90.1512 & 0.0153 & 0.0065 & 0.0138 & 0.0170 & 0.0102 & 103 \\
\hline 4 & 54 & 54 & 3 & 90.1513 & 0.0136 & 0.0056 & 0.0124 & 0.0161 & 0.0134 & 105 \\
\hline 5 & 60 & 43 & 4 & 90.1514 & 0.0135 & 0.0050 & 0.0125 & 0.0154 & 0.0166 & 114 \\
\hline 6 & 64 & 36 & 5 & 90.1513 & 0.0124 & 0.0046 & 0.0115 & 0.0148 & 0.0197 & 114 \\
\hline 7 & 63 & 31 & 6 & 90.1512 & 0.0122 & 0.0043 & 0.0114 & 0.0149 & 0.0229 & 119 \\
\hline 10 & 73 & 21 & 9 & 90.1514 & 0.0112 & 0.0035 & 0.0106 & 0.0139 & 0.0325 & 128 \\
\hline
\end{tabular}




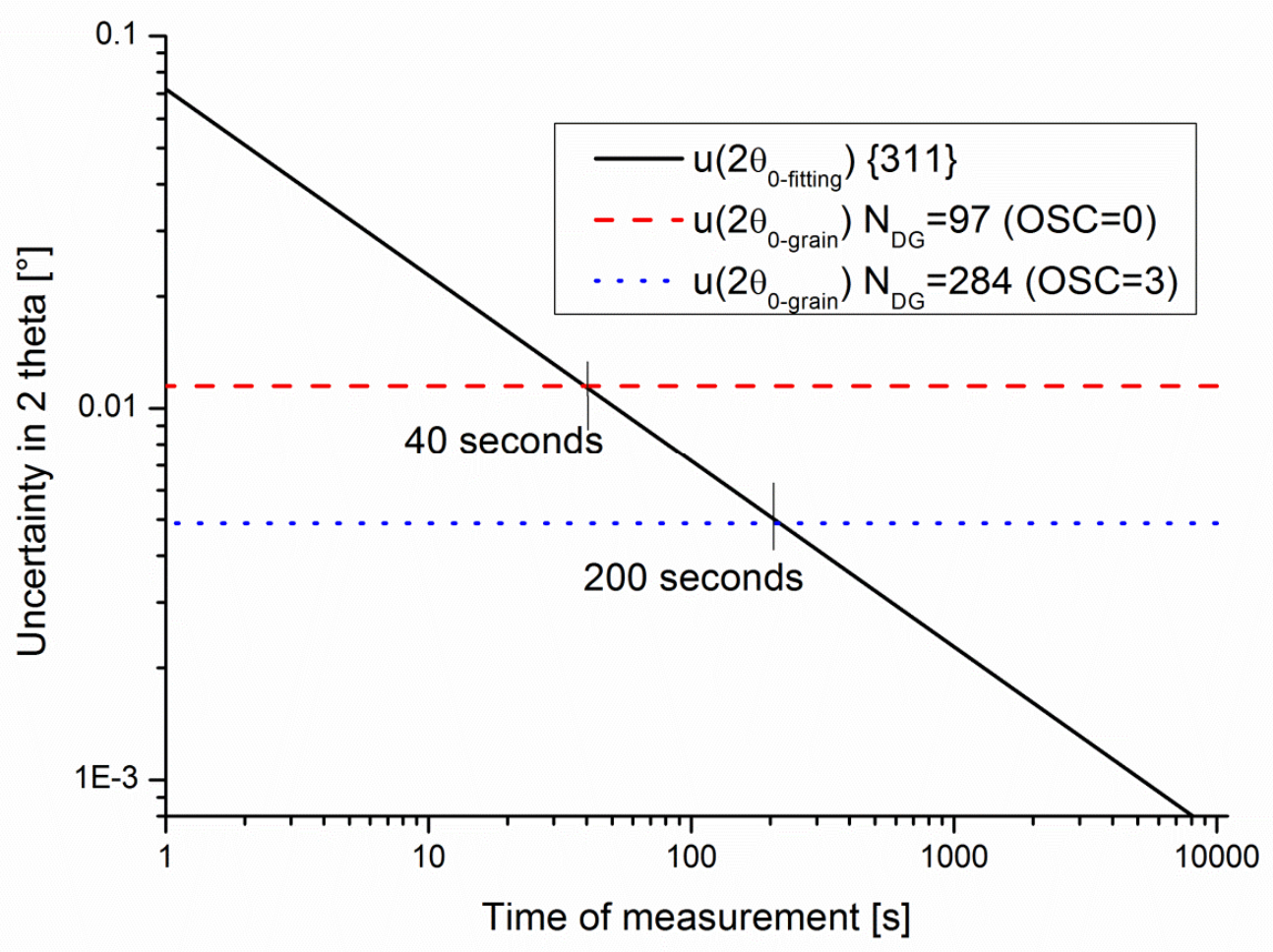

Figure 4. This shows the fitting uncertainty as a function of time for the $\{311\}$ reflection for the black parent reference coupon on $\mathrm{E} 3$. The grain size uncertainty for $\mathrm{OSC}=0$ and $\mathrm{OSC}=3$ are also shown. Eq. 6 (b) can be used to calculate the total uncertainty. For this particular experimental set-up and specimen, it takes 40 seconds to get to a fitting uncertainty of about $\pm 0.01^{\circ}$. If one does not oscillate $(\mathrm{OSC}=0)$ the total uncertainty cannot get any lower as one has a value of $N_{D G} \approx 97$ which places an upper bound of the value of $u\left(2 \theta_{0 \text {-grain }}\right) \approx \pm 0.0115^{\circ}$. It takes 200 seconds to get to a fitting uncertainty of about $\pm 0.005^{\circ}$. If one oscillates $(\mathrm{OSC}=3$ ) the total uncertainty cannot get any lower as one has a value of $N_{D G} \approx 284$ which places an upper bound of the value of $u\left(2 \theta_{0 \text {-grain }}\right)$ $\approx \pm 0.0049^{\circ}$. 


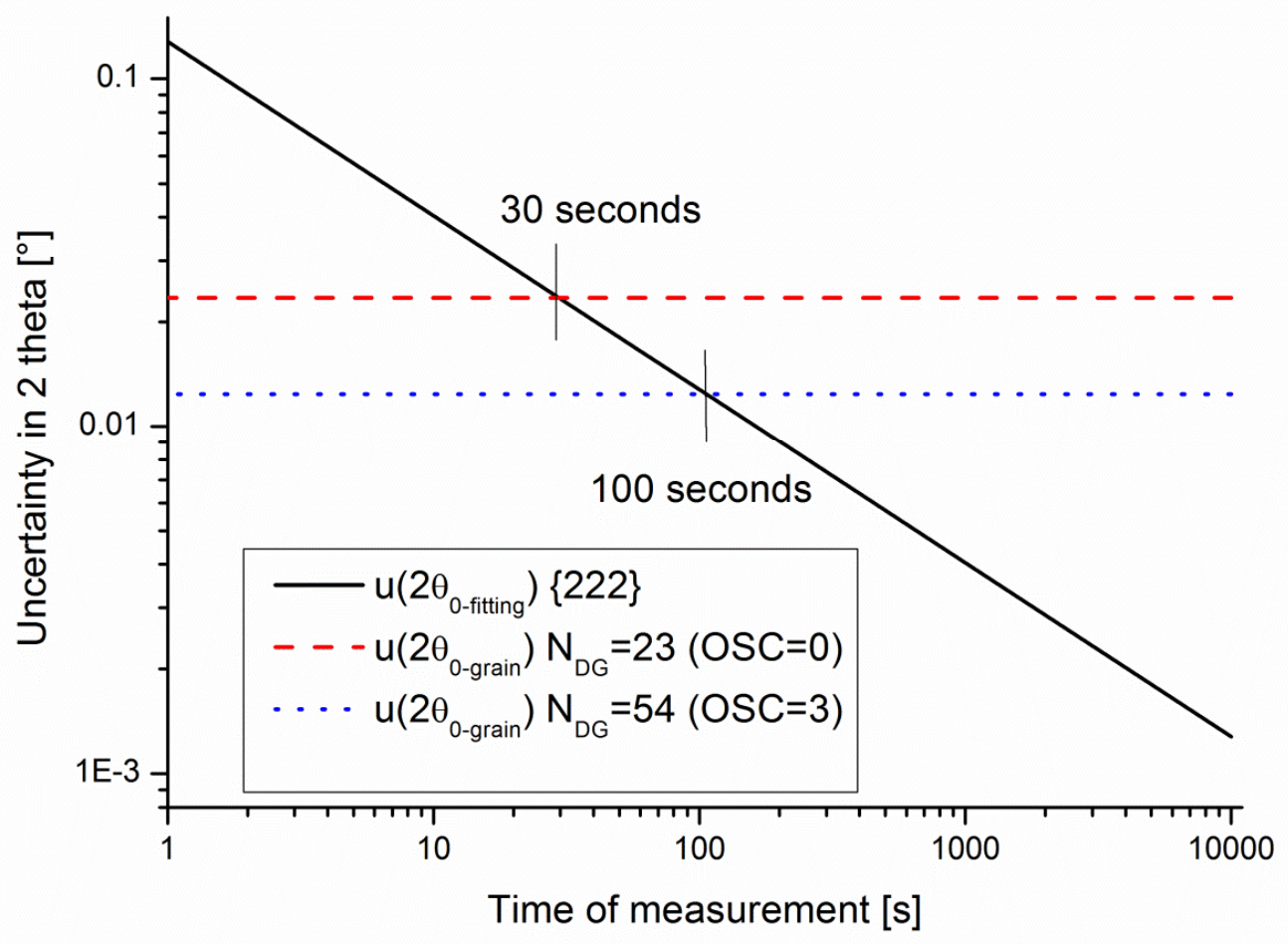

Figure 5. shows the fitting uncertainty as a function of time $\left\{\begin{array}{ll}2 & 2\end{array} 2\right\}$ reflection for the black parent reference coupon on $\mathrm{E} 3$. The grain size uncertainty for $\mathrm{OSC}=0$ and $\mathrm{OSC}=3$ are also shown. Eq. 6(b) can be used to calculate the total uncertainty. For this particular experimental set-up and specimen, it takes 30 seconds to get to a fitting uncertainty of about $\pm 0.025^{\circ}$. If one does not oscillate $(O S C=0)$ the total uncertainty cannot get any lower as one has a value of $N_{D G} \approx 23$ which places an upper bound of the value of $u\left(2 \theta_{0 \text {-grain }}\right) \approx \pm 0.0235^{\circ}$. It takes 100 seconds to get to a fitting uncertainty of about $\pm 0.012^{\circ}$. If one oscillates $(\mathrm{OSC}=3)$ the total uncertainty cannot get any lower as one has a value of $N_{D G} \approx 54$ which places an upper bound of the value of $u\left(2 \theta_{0 \text {-grain }}\right)$ $\approx \pm 0.0124^{\circ}$ 
In a totally separate measurement the TG4 reference SET $Y$ was also measured using different gauge volumes. Gauge volumes from $3 \times 3 \times 2 \mathrm{~mm}^{3}$ to $8 \times 8 \times 2 \mathrm{~mm}^{3}$ were used for the parent material (one side coloured black), top $3 \mathrm{~mm}$ of the weld fusion region (one side coloured red) and bottom $3 \mathrm{~mm}$ of the weld fusion region (one side coloured green). The number of apparent detected diffracting grains was calculated from Eq. 8. The values obtained for $u\left(2 \theta_{0 \text {-grain }}\right)$ was obtained from Eq. $6(\mathrm{~b})$. Larger gauge volumes were used compared to the previous measurement and the multiplicity ratio of $\mathrm{m}^{311} / \mathrm{m}^{222}$ is appears to be closer 3 , and this is approximately reflected in the number of detected diffracting grains.

Figure 6 shows that the results from the reference coupons approximately follow the trend of the model: $u\left(2 \theta_{0-\text { grain }}\right) \approx \frac{0.5 * S D_{\text {Gauss }}}{\left(N_{D G}\right)^{1 / 2}}$.

The graph shows that in order to trust a fitting uncertainty of $\pm 0.01^{\circ}$ one needs to have more than 100 detected diffracting grains when using a Bragg peak with, for example, a $S D_{\text {gauss }}=0.2^{\circ}\left(\mathrm{FWHM}\right.$ of $\left.0.47^{\circ}\right)$. The black coupon $\left\{\begin{array}{lll}3 & 1 & 1\end{array}\right\}$ comes the closest to meeting this criterion, although it would be better if the specimens were oscillated during measurement.

The grain size has been estimated from Eq. 11 for all gauge volumes less than $5 \times 5 \times$ $2 \mathrm{~mm}^{3}$. The typical values were $42-109 \mu \mathrm{m}$ for the parent material, $137-174 \mu \mathrm{m}$ for the bottom of the weld and 175-276 $\mu \mathrm{m}$ for the top of the weld.

Measuring the red coupon with a small gauge volume became increasing more difficult because of the lack of detected diffracting grains.

As seen in the previous coupon measurement, if one is to rely on the fitting uncertainty only, the uncertainty due to grain size has to be supressed. It is recommended that the upper bound of the value of $u\left(2 \theta_{\text {grain }}\right)$ should be no more than $\approx \pm 0.015^{\circ}$. From Eq. 10 it can be seen that for a FWHM of $0.6^{\circ}$ the number of detected diffracting grains $N_{D G} \approx 72$. For a FWHM $=0.5^{\circ}, 0.4^{\circ}$ and $0.3^{\circ}$, then $N_{D G} \approx 50,32$ and 18 respectively, The smaller the FWHM of the peak, the smaller the value of $N_{D G}$ needed [27].

The time of measurement should not be too long otherwise the fitting uncertainty will be an underestimate of the total uncertainty. In order to increase the value of $N_{D G}$, oscillation of the specimen is recommended. If the value of $N_{D G}$ needed cannot be achieved then the method described by Holden et. al. [28] is recommended (if it is possible), where the specimen is measured and then rotated 180 degrees and measurement at the same place again and the result is averaged. 


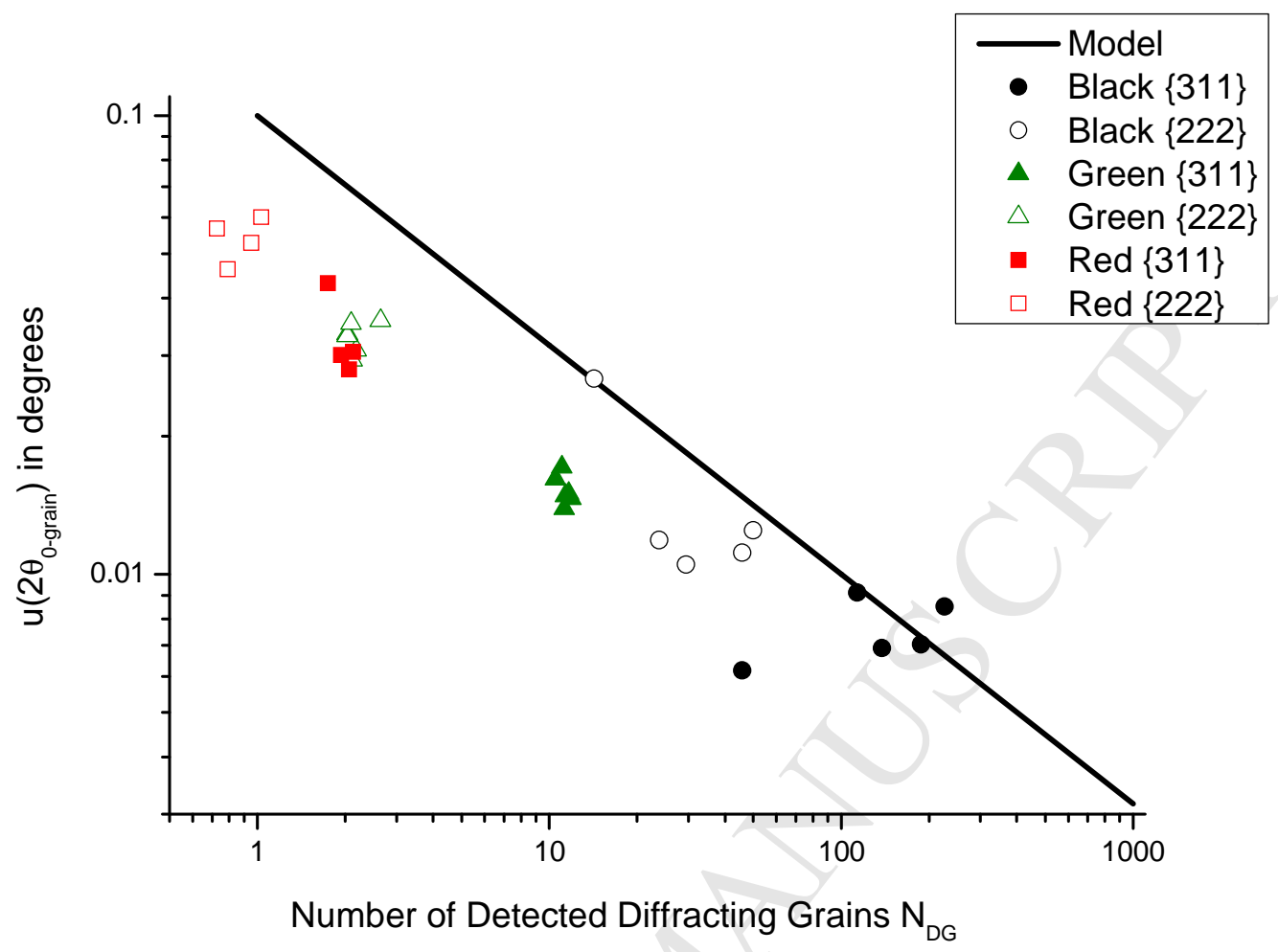

Figure 6. A comparison of the model with results of the three reference coupons. The results show the relation of number of detected diffracting grains with the random uncertainty due to the grain size. The influence of multiplicity can also be seen, where using the $\left\{\begin{array}{lll}2 & 2 & 2\end{array}\right\}$ reflection results in have approximately three times less detected diffracting grains than $\left\{\begin{array}{llll}3 & 1 & 1\end{array}\right\}$.

\subsection{The $D$ line results}

In this section a comparison of data from different laboratories is presented. The TG4 specimens were oscillated in omega ( $\omega$-axis) in the single peak neutron diffraction experiments, meaning that more grains are observed on the detector, thus reducing the impact of the grain-size effect. The synchrotron data used 5 peaks for the strain measurement and the data is recorded for larger parts of the Debye-Scherrer-rings however there was a smaller effective gauge volume $(0.014 \mathrm{~mm} \times 0.014 \mathrm{~mm}$ beam size plus a linear oscillation of $\Delta z= \pm 2 \mathrm{~mm}$ ).

The D9 line is completely within the parent material of the specimen. This is thus expected to show up the least grain size effect as the grain size appears smaller than in the weld region, as seen in the reference coupon specimens in the previous section. Table 5 shows the experimental parameters for the neutron measurements. In the 
comparisons the data from the $3 \times 3 \times 2 \mathrm{~mm}^{3}$ gauge volume of the reference specimen data from the previous section will be used. The data has been collected over a period of time, for instance, the HZB measurements on the TG4 plate were made in 2009 and used a slit set-up. The detector was further away than with the 2013 measurements on the coupons (which used a radial collimator as a secondary optic), hence the difference in the $D_{H}$ values. The monochromator set up was also different, hence the slightly different $S D_{\text {Gauss }}$ values. The larger $S D_{\text {Gauss }}$ values for the $\left\{\begin{array}{ll}2 & 2\end{array} 2\right\}$ reflection compared to the $\left\{\begin{array}{lll}3 & 1 & 1\end{array}\right\}$ reflection is due to an angular resolution difference of the monochromator as a function of $2 \theta$.

Table 5. Experimental parameters for the neutron measurements.

\begin{tabular}{|c|c|c|c|c|c|}
\hline Parameter & HZB ref & HZB ref & FRM II (a) & FRM II (b) & HZB \\
\hline Date & 2013 & 2013 & 2009 & 2010 & 2009 \\
\hline Sample & Ref SET Y & Ref SET Y & TG4 3-1A plate & TG4 3-1A plate & TG4 3-1A plate \\
\hline Ref & SET Y & SET Y & SET Z & SET Z & SET W \\
\hline$\{h \mathbf{k I}\}$ & $\left\{\begin{array}{lll}2 & 2 & 2\end{array}\right.$ & $\{3 \quad 11\}$ & $\{3111\}$ & $\{311\}$ & $\left\{\begin{array}{lll}3 & 1 & 1\end{array}\right\}$ \\
\hline$m^{h k l}$ & 8 & 24 & 24 & 24 & 24 \\
\hline $\boldsymbol{\omega}_{M}\left[^{\circ}\right]$ & 1.2 & 1.2 & 1.2 & 1.2 & 1.2 \\
\hline$\eta_{M}\left[^{\circ}\right]$ & 1.2 & 1.2 & 1.2 & 1.2 & 1.2 \\
\hline$D_{H}\left[^{\circ}\right]$ & 15 & 15 & 10 & 12 & 12 \\
\hline $\mathrm{OSC}\left[^{\circ}\right]$ & 0 & 0 & 6 & 8 & 10 \\
\hline Optics $\left[\mathrm{mm}^{3}\right]$ & $3 \times 3 \times 2$ & $3 \times 3 \times 2$ & $2 \times 2 \times 2$ & $3 \times 3 \times 2.1$ & $3 \times 3 \times 3$ \\
\hline $2 \theta\left[^{\circ}\right]$ & 91.6 & 86.6 & 92.5 & 92.9 & 86.5 \\
\hline$g v\left[\mathrm{~mm}^{3}\right]$ & 18 & 18 & 8.01 & 18.92 & 27.05 \\
\hline$P$ & 0.00377 & 0.01131 & 0.04691 & 0.07065 & 0.08601 \\
\hline$S D_{\text {Gauss }}\left[^{\circ}\right]$ & 0.215 & 0.187 & 0.246 & 0.265 & 0.177 \\
\hline
\end{tabular}

All neutron measurements used two-dimensional ${ }_{3} \mathrm{He}$ position sensitive detectors (PSDs). These are multi-wire detectors with a delay time encoding with data binning of $256 \times 256$ 'pixels'. The one used by HZB was developed by DENEX, Germany, with a spatial resolution of about $1.2 \times 1.2 \mathrm{~mm}^{2}$ with an active area of $300 \times 300 \mathrm{~mm}^{2}$. The detector used for the FRM II (a) measurements was developed by EMBL (Grenoble, France) with a spatial resolution of about $1.5 \times 1.5 \mathrm{~mm}^{2}$ and an active area of $200 \times$ $200 \mathrm{~mm}^{2}$. The detector used for the FRM II (b) measurements was developed by Mirrotron Hungary, and it has a nominal spatial resolution of $2 \times 2 \mathrm{~mm}^{2}$ with an active area of $300 \times 300 \mathrm{~mm}^{2}$.

Many measurements were made in NeT-TG4 and this gave a good opportunity to calculate a robust average of all the measurements. This meant that with the data sets presented here, one could take away the robust average and study the residuals to calculate the actual systematic offsets and random uncertainties (which contains the grain size contribution as well as fitting uncertainties). It should be noted that the robust 
average contained measurements on other nominally the same NeT-TG4 specimens, i.e. the $1-1 \mathrm{~A}$ and $2-1 \mathrm{~A}$ specimens.

Figure 7 shows a comparison of robust averages with the HZB E3 data for the Longitudinal stress D2 and D9 lines. One can see that the D9 data agrees well with the average and the quoted uncertainties appear also to be reasonable. For the D2 line the measurement points between $z=-40$ and $40 \mathrm{~mm}$ are within the weld material. The scatter of the data is clearly more than that of the D9 line (which is completely in parent material). In addition there appears to be a systematic offset in the weld region, where the stress values appear to be underestimated. The data points outside of the weld on the D2 line (which are in the parent material) agree well with the average.

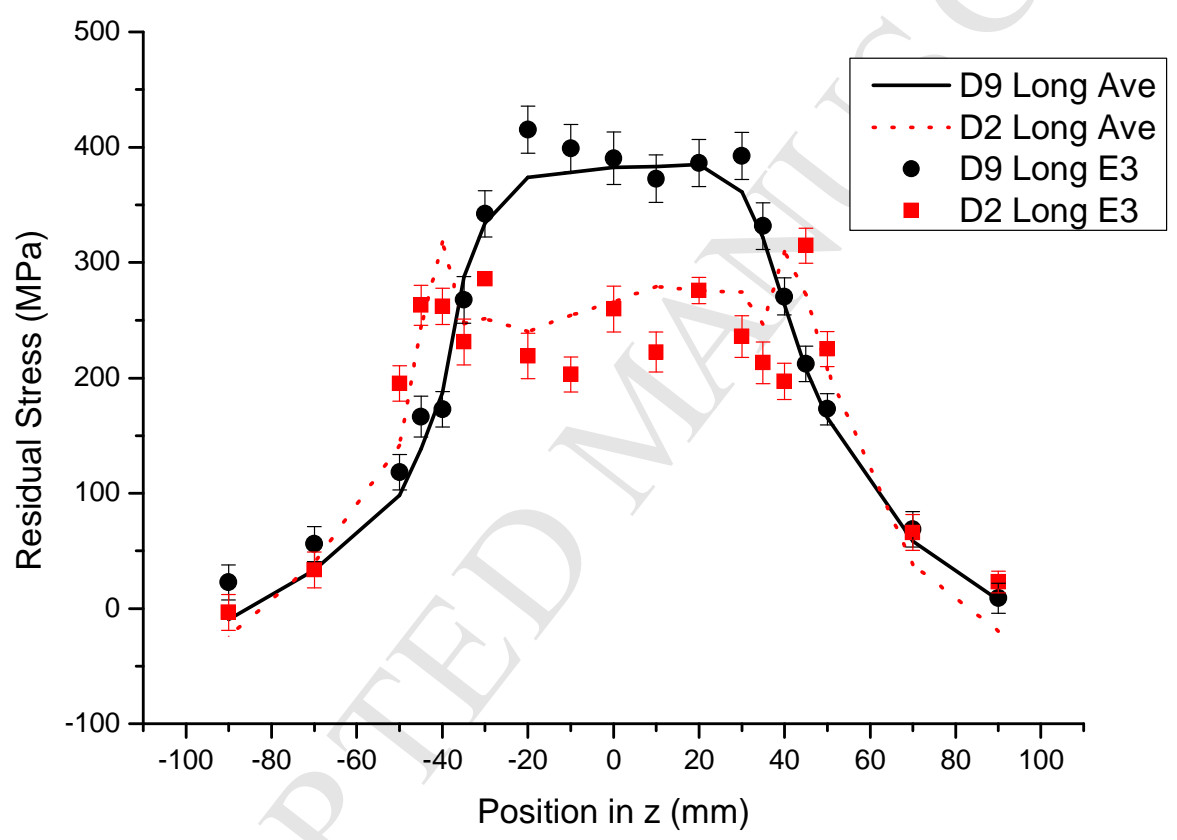

Figure 7. A comparison of robust averages with the HZB E3 data for the D2 and D9 lines in the longitudinal stress direction.

In order to discriminate against outliers in the data, after taking away the appropriate robust average from each data set, the residuals were arranged equidistantly in magnitude order (see Figure 8). Subsequently after scaling the abscissa: $-100 \%$ to $100 \%$, a linear fit was made between $\pm 68.28 \%$, corresponding to \pm 1 standard deviation (between the two vertical lines in Figure 8). This linear fit gives simultaneously the systematic offset and the total random uncertainty from the gradient. These linear fitting calculations will be referred to as R-fits (Residual fits) from now on. Of the total 19 points in each direction of the D9 line, 13 points lie within the first standard deviation. For the D5 and D2 lines, the measurement points between $z=-40$ and $40 \mathrm{~mm}$ are within the weld material and only these were considered to estimate the systematic and 
random uncertainties. This meant that out of a total number of points of 11 in the weld region, 7 points laid within the first standard deviation (see Figure 8).

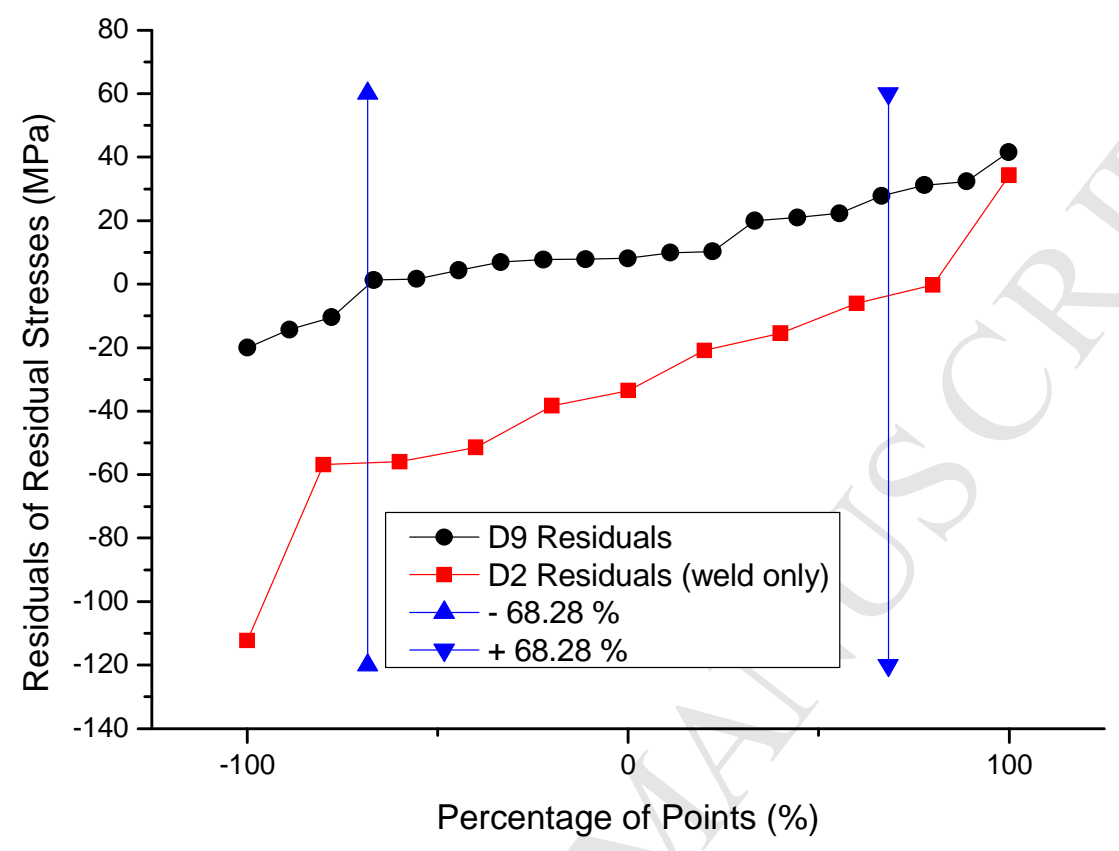

Figure 8. Residuals arranged in magnitude order. Linear fits were applied to the points between $\pm 68.28 \%$, corresponding to \pm 1 standard deviation.

The gradient of the longitudinal stress D9 and D2 lines fits are $0.185 \mathrm{MPa} / \%$ and $0.427 \mathrm{MP} / \%$ respectively, multiplying these values by $68.28 \%$ gives $12.64 \mathrm{MPa}$ and 29.12 MPa. These values are an estimate of the underlying random uncertainty. The average quoted fitting uncertainties are $\pm 17.79 \mathrm{MPa}$ and $\pm 17.10 \mathrm{MPa}$ for D9 and D2 respectively. The peaks were fitted using a Gaussian function with a linear background and so the fitting uncertainty could be over-estimated as was seen in Table 1. A different program was used back in 2009 to evaluate this TG4 plate data called TVtueb [26] which only had a Gaussian function with a linear background option. The D2 line has a much larger random uncertainty, much larger than the quoted fitting uncertainty. This is due to the grain size contribution of random uncertainty in the weld region. The corresponding systematic offsets are $+11.45 \mathrm{MPa}$ for the D9 line and $-31.65 \mathrm{MPa}$ for the D2 line (in the weld region). The R- fit analysis was performed for all $D$ lines and averaged over the three stress directions.

Using the model in Eq. 10 and propagating the numbers through Eq. 7 and then Eq. 3 (Assuming $u\left(\varepsilon_{x x}\right)=u\left(\varepsilon_{y y}\right)=u\left(\varepsilon_{z z}\right)$ ), one can estimate the corresponding strain and stress uncertainties due to grain size. The elastic moduli values used are $E_{311}=183.6 \mathrm{GPa}$, 
$v_{311}=0.306, E_{222}=209.4 \mathrm{GPa}$, and $v_{222}=0.278$ [12]. Table 6 shows the values one should get using these equations for the parent material. A distinction is made between the reference $u\left(2 \theta_{0 \text {-grain }}\right)$ and non-reference values $u\left(2 \theta_{\text {grain }}\right)$ for $\mathrm{Eq}$. 7 . These values have to be divided by two before placing through Eq. 7. The fitting values are set to zero, as we are only considering the extra random uncertainty due to grain size: $u\left(2 \theta_{0}\right.$. fitting $)=u\left(2 \theta_{\text {fitting }}\right)=0$. A grain size of $0.09 \mathrm{~mm}(90 \mu \mathrm{m})$ was assumed for the parent material. One can see the influence of multiplicity and $P$ value on the uncertainty values, i.e. the higher the $P$ and multiplicity values, the less the value of uncertainty. The larger gauge volume on the HZB E3 measurements also decreases the uncertainty values.

Table 6. Calculation of strain and stress random uncertainties due to grain size, assuming a grain size of $0.09 \mathrm{~mm}(90 \mu \mathrm{m}) \cdot u\left(2 \theta_{0 \text {-fiting }}\right)=u\left(2 \theta_{\text {fitting }}\right)=0$

\begin{tabular}{|c|c|c|c|}
\hline Sample & $\begin{array}{l}\text { Black Coupon } \\
\text { Reference SET Y }\end{array}$ & $\begin{array}{l}\text { Black Coupon } \\
\text { Reference SET Y }\end{array}$ & $\begin{array}{c}\text { HZB D9 } \\
\text { TG4 3-1A }\end{array}$ \\
\hline$\{$ h k I $\}$ & $\left\{\begin{array}{lll}2 & 2 & 2\end{array}\right\}$ & $\{311\}$ & $\left\{\begin{array}{lll}3 & 1 & 1\end{array}\right\}$ \\
\hline $\mathrm{m}^{\mathrm{nkI}}$ & 8 & 24 & 24 \\
\hline $\mathbf{P}$ & 0.00377 & 0.01131 & 0.08601 \\
\hline $\mathrm{gv}\left[\mathrm{mm}^{3}\right]$ & 18 & 18 & 27.05 \\
\hline $\mathrm{SD}_{\text {Gauss }}\left[{ }^{\circ}\right]$ & 0.215 & 0.187 & 0.177 \\
\hline $\mathbf{u}\left(\mathbf{2 \theta _ { 0 - \text { grain } } ) [ { } ^ { \circ } ]}\right.$ & 0.0111 & 0.0056 & - \\
\hline $\mathbf{u}\left(2 \boldsymbol{\theta}_{\text {grain }}\right)\left[{ }^{\circ}\right]$ & - & $y$ & 0.0016 \\
\hline $\begin{array}{c}\mathrm{u}(\varepsilon)[\mu \mathrm{m}] \\
(\mathrm{Eq} .7)\end{array}$ & 94 & 52 & 15 \\
\hline $\begin{array}{c}\mathrm{u}(\sigma)[\mathrm{MPa}] \\
(\mathrm{Eq} .3) \text { assuming } \\
\mathrm{u}\left(\varepsilon_{\mathrm{xx}}\right)=\mathrm{u}\left(\varepsilon_{\mathrm{yy}}\right)=\mathrm{u}\left(\varepsilon_{\mathrm{zz}}\right)\end{array}$ & 29 & 15 & 4 \\
\hline
\end{tabular}

A comparison can now be made with the random uncertainties estimated from the $\mathrm{R}$ fits. These R-fits also include the fitting uncertainty and so a nominal fitting uncertainty of $15 \mathrm{MPa}$ has been added to the model (using root mean squares) as this was a typical quoted and actual uncertainty value for the FRMII and HZB measurements in the parent material (see Table 10). Table 7 shows the values of the model assuming a grain size of $0.09 \mathrm{~mm}(90 \mu \mathrm{m})$, Table 8 , a grain size of $0.180 \mathrm{~mm}(180 \mu \mathrm{m})$ and Table 9 , a grain size of $0.260 \mathrm{~mm}(260 \mu \mathrm{m})$. The data has been plotted out in Figure 9 . 
Table 7. Comparison of model stress random uncertainties, assuming a grain size of $0.09 \mathrm{~mm}(90 \mu \mathrm{m})$ with random uncertainties estimated from the R-fits.

\begin{tabular}{c|c|c|c|c} 
Sample & $\boldsymbol{P}$ & $\begin{array}{c}\text { D9 Model } \\
\text { [MPa] }\end{array}$ & $\begin{array}{c}\text { Including fitting } \\
\text { uncertainty of 15 [MPa] }\end{array}$ & $\begin{array}{c}\text { Random uncertainty } \\
\text { from R-fits [MPa] }\end{array}$ \\
\hline Black coupon \{2 2 2\} & 0.00377 & 28.7 & 32.4 & 31.0 \\
\hline Black coupon \{3 1 1\} & 0.01131 & 15.3 & 21.5 & 19.7 \\
\hline FRM II (a) D9 & 0.04691 & 13.4 & 20.1 & 15.8 \\
\hline FRM II (b) D9 & 0.07065 & 7.6 & 16.8 & 22.8 \\
\hline HZB E3 D9 & 0.08601 & 4.3 & 15.6 & 14.8
\end{tabular}

Table 8. Comparison of model stress random uncertainties, assuming a grain size of $0.180 \mathrm{~mm}(180 \mu \mathrm{m})$ with random uncertainties estimated from the R-fits.

\begin{tabular}{c|c|c|c|c} 
Sample & $\boldsymbol{P}$ & $\begin{array}{c}\text { D5 Model } \\
\text { [MPa] }\end{array}$ & $\begin{array}{c}\text { Including fitting } \\
\text { uncertainty of 15 [MPa] }\end{array}$ & $\begin{array}{c}\text { Random uncertainty } \\
\text { from R-fits [MPa] }\end{array}$ \\
\hline Green coupon \{2 2 2\} & 0.00377 & 84.0 & 85.4 & 95.8 \\
\hline Green coupon \{3 1 1 & 0.01131 & 43.4 & 45.9 & 40.7 \\
\hline FRM II (a) D5 & 0.04691 & 37.9 & 40.8 & 52.5 \\
\hline FRM II (b) D5 & 0.07065 & 21.5 & 26.2 & 29.1 \\
\hline HZB E3 D5 & 0.08601 & 12.2 & 19.3 & 18.2
\end{tabular}

Table 9. Comparison of model stress random uncertainties, assuming a grain size of $0.260 \mathrm{~mm}(260 \mu \mathrm{m})$ with random uncertainties estimated from the R-fits.

\begin{tabular}{c|c|c|c|c} 
Sample & $\boldsymbol{P}$ & $\begin{array}{c}\text { D2 Model } \\
\text { [MPa] }\end{array}$ & $\begin{array}{c}\text { Including fitting } \\
\text { uncertainty of 15 [MPa] }\end{array}$ & $\begin{array}{c}\text { Random uncertainty } \\
\text { from R-fits [MPa] }\end{array}$ \\
\hline Red coupon \{2 2 2\} & 0.00377 & 145.9 & 146.7 & 141.6 \\
\hline Red coupon \{3 1 1 & 0.01131 & 75.3 & 76.8 & 76.9 \\
\hline FRM II (a) D2 & 0.04691 & 65.9 & 67.5 & 49.7 \\
\hline FRM II (b) D2 & 0.07065 & 37.4 & 40.2 & 39.2 \\
\hline HZB D2 & 0.08601 & 21.1 & 25.9 & 33.9
\end{tabular}




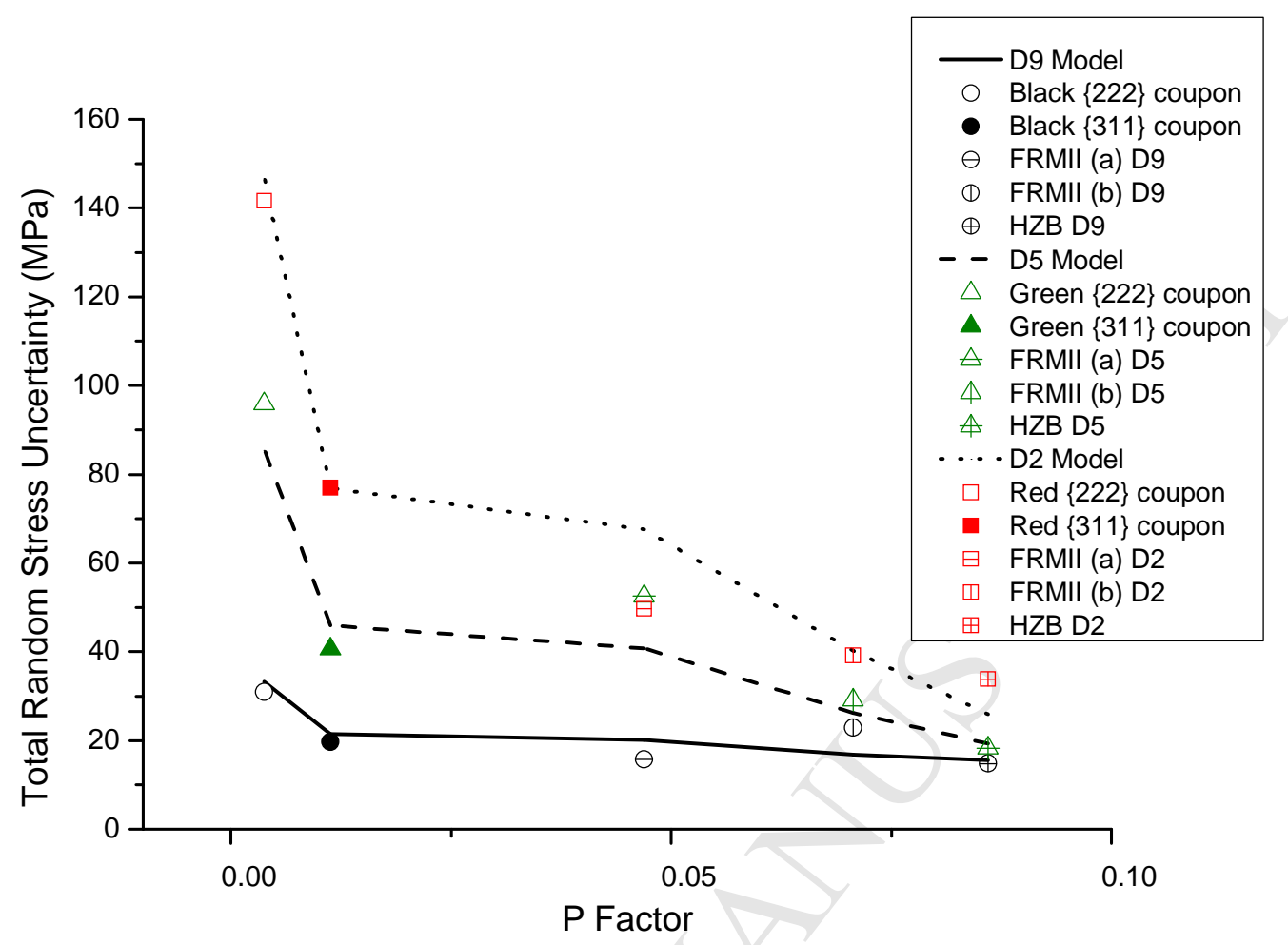

Figure 9. Comparison of model stress random uncertainties with the random uncertainties estimated from the R-fits.

Figure 9 show the model results against the actual random uncertainties obtained from the $R$ fits. The agreement with the model is very good. The effect of multiplicity, gauge volume, $\mathrm{P}$ factor, grain size and the insignificance of quoted stress uncertainties derived from the fitting uncertainties for this example can all be seen. This also shows that the green reference coupon is appropriate for the D5 line and the red coupon is appropriate for the D2 line. Even for the weld material after oscillation, the fitting uncertainty is still not enough to represent the total actual random uncertainty of the measurement. Table 10 shows a comparison of the average quoted stress uncertainties with the actual random stress uncertainties. The uncertainties due to the reference measurements are also included in the quoted uncertainties but these are much less than the sample measurement uncertainties and do not make a large difference when taken away using root mean squares. Table 11 shows the systematic offsets derived from the R-fits.

In general it can be seen that for the weld region, the fitting uncertainty is inadequate for estimating stress uncertainty. With increased gauge volume and oscillation the values become closer, especially in the parent material, where the grain size is smaller. The $\mathrm{JRC/ESRF}$ quoted uncertainties are very good and even overestimate the actual stress uncertainty. The synchrotron data used 5 peaks for the strain measurement and the data is recorded for larger parts of the Debye-Scherrer-rings, although with a smaller effective gauge volume. It was the only data set that included $5 \%$ uncertainty of the 
Poisson ratio and taking this into account the quoted uncertainties are even closer to the actual random uncertainty. As a rule of thumb, stress uncertainties of greater than $40 \mathrm{MPa}$ in steel are too high and efforts to reduce the uncertainty should be implemented.

Table 10. A comparison of quoted stress uncertainties compared to the actual random stress uncertainties derived from the R-fits.

\begin{tabular}{c|c|c|c|c|c|c|c|c}
$\begin{array}{c}\text { D } \\
\text { line }\end{array}$ & \multicolumn{2}{|c|}{ FRM II (a) [MPa] } & \multicolumn{2}{c|}{ FRM II (b) [MPa] } & \multicolumn{2}{c}{ HZB [MPa] } & \multicolumn{2}{c}{ JRC/ESRF [MPa] } \\
\hline & Quoted & Actual & Quoted & Actual & Quoted & Actual & Quoted & Actual \\
\hline D9 & 26 & $\mathbf{1 6}$ & 15 & $\mathbf{2 3}$ & 15 & $\mathbf{1 5}$ & 35 & $\mathbf{3 0}$ \\
\hline D5 & 25 & $\mathbf{5 3}$ & 16 & $\mathbf{2 9}$ & 17 & $\mathbf{1 8}$ & 42 & $\mathbf{3 7}$ \\
\hline D2 & 30 & $\mathbf{5 0}$ & 16 & $\mathbf{4 0}$ & 16 & $\mathbf{3 4}$ & 38 & $\mathbf{2 6}$
\end{tabular}

Table 11. Systematic uncertainties derived from the R-fits.

\begin{tabular}{c|c|c|c|c|c} 
D line & $\begin{array}{c}\text { FRM II (a) } \\
\text { [MPa] }\end{array}$ & $\begin{array}{c}\text { FRM II (b) } \\
\text { [MPa] }\end{array}$ & $\begin{array}{c}\text { HZB } \\
\text { [MPa] }\end{array}$ & $\begin{array}{c}\text { JRC/ESRF } \\
\text { [MPa] }\end{array}$ & $\begin{array}{c}\text { JRC/ESRF } \\
\text { plus normal } \\
\text { stress } \\
\text { [MPa] }\end{array}$ \\
\hline D9 & -21 & 2 & 5 & -13 & 2 \\
\hline D5 & -38 & -21 & 0 & -59 & -26 \\
\hline D2 & -23 & -23 & -27 & -20 & 18
\end{tabular}

The systematic uncertainties in general are negative in the weld region, which may suggest that the TG4 sample 3-1A may have less stress in this region than the other samples, as the robust average was averaged over different specimens. In general though the systematic uncertainty offset decreases with an increase in $\mathrm{P}$ factor/gauge volume and the estimation is better with the HZB measurements, which used the largest oscillation and gauge volume. It should be noted that different sets of reference coupon specimens were used for each measurement (see Table 5). The JRC/ESRF measurements assumed zero stress in the normal direction for calculating stress in the longitudinal and transverse directions. From the robust averages it can be seen that this is not the case, the normal stress is slightly positive in the normal stress direction in the weld, a triaxial stress distribution exists. These values have been added to the systematic uncertainty in the last column of Table 12 to show that the underlying estimation of systematic uncertainty is good. These values agree well with the FRM II (b) values in D9 and D5, where both sets of measurements used reference coupon SET Z. The reference coupons for the 2009 E3 TG4 3-1A measurement (SET W) were rotated continuously around a large $\omega$ value and summed in a single file. The systematic offset estimation is very good (i.e. close to zero) for the D9 and D5 lines. The D2 line is negative, which may either be because the overall stress in the weld region for the 3-1A specimen is lower or there is still an issue with the correct systematic offset estimation. 
Figure 10 shows an estimation of grain size from the synchrotron data measured by JRC at the ESRF. The values agree well with the E3 reference coupon measurements grain size estimations. The coupon measurements showed typical values of $42-109 \mu \mathrm{m}$ for the parent material ( $\mathrm{y}=8$ to $17 \mathrm{~mm}$ in Figure 10), $137-174 \mu \mathrm{m}$ for the bottom of the weld $(y=5 \mathrm{~mm}$ in Figure 10$)$ and $175-276 \mu \mathrm{m}$ for the top of the weld $(y=2 \mathrm{~mm}$ in Figure 10). It has to be noted, that the present calculation did not take into account the grain shape, i.e. the aspect ratio is taken as 1 . Therefore, it is not unlikely that columnar grains in the weld zone are actually longer than $200 \mu \mathrm{m}$. The grain size estimations for the model (which fitted the data best) in Tables 7, 8 and 9 are $90 \mu \mathrm{m}, 180 \mu \mathrm{m}$ and $260 \mu \mathrm{m}$ respectively. It should be noted however that there are uncertainties associated with all parameters used in the model. The gauge volume is probably the most difficult to estimate as the divergence of the neutron beam is often slightly larger than the nominal volume set by the beam defining optics.

One set of neutron data for the $D$ lines used a radial oscillating collimator for the secondary collimation of the neutrons. The stress results from that measurement agreed well with the other two sets of neutron data which utilized a slit for the secondary collimation. When using slits however, the secondary slit should not be more than 60 $\mathrm{mm}$ away from the centre of the gauge volume as standard good practice [27]. A slit suffers from aberrations similar to the pin-hole camera effect if the distances are larger. A radial oscillating collimator eliminates this effect and can be used at a larger distance away from the centre of the gauge volume. The use of a secondary slit can also alter the shape of the peak, especially if one measures a relatively broad peak with a small slit width. The detected tails of the peak can be attenuated, causing the peak to be less Gaussian in shape and thus the fitting program over estimates the uncertainty. This maybe the cause of the over-estimation of quoted fitting uncertainty for the parent material for the FRM II (a) measurement (see Table 10, D9 results).

Grain size estimation from the microscopic studies at the Open University in the parent material region provided values of $75 \pm 12 \mu \mathrm{m}$, over grains without twins and $67 \pm 10 \mu \mathrm{m}$, over grains with twins, each average calculated over 20 values. This agrees well with the JRC/ESRF estimation $83 \pm 4 \mu \mathrm{m}$ (from 11 values, $\mathrm{y}=8$ to $17 \mathrm{~mm}$, in Figure 10). These values also agree well with the E3 reference coupon measurements grain size estimations in Tables 3 and 4.

A separate study in the parent material of the TG4 specimen made by JRC (made at a different location in the specimen) gave a result of $92 \pm 9 \mu \mathrm{m}$ (with a range of values 78$106 \mu \mathrm{m})$. This agrees well with the grain size estimations from reference specimens. This indicates that the grain size does vary slightly from place to place in the parent material. 


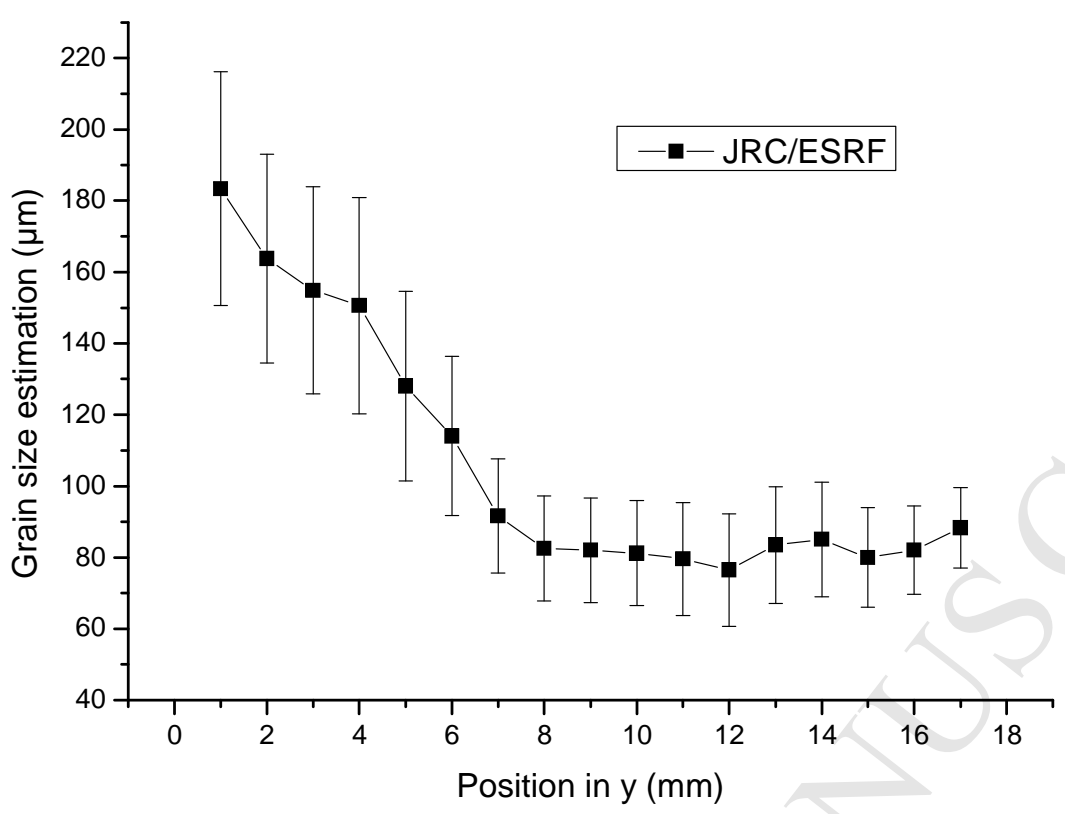

Figure 10. Estimation of grain size from the synchrotron data measured by JRC at the ESRF.

\section{Conclusions}

By presenting sets of diffraction data, obtained for an identical weld specimen on different strain scanning instruments, it has been demonstrated how grain size can influence the accuracy of a measurement. The traditional method of propagating the uncertainty of a function fit (normally a Gaussian shape on a linear background) relies on good counting statistics to be accurate enough. Counting statistics in turn relies on there being enough diffracting grains being detected to approximate to good counting statistics (and a good Gaussian shape of the data), however when there are not enough grains in the diffracting volume considered, the analysis is inadequate.

Measurement of reference coupons is useful in that it does not only give a strain free reference value but also gives an indication of how the instrument and material behaves while using only little time for the measurement (because of the short neutron path length compared to the corresponding larger specimen). Numerous tests can be carried out simply by rotating and measuring the specimen multiple times, for example in steps of 1 or 2 degrees. A comparison of the average fitting uncertainty with the standard deviation of the whole data set is a good way to check for uncertainty due to grain size. In terms of time the fitting uncertainty should improve (decrease in value), but the 
standard deviation of the whole data set approaches a certain underlying value if there is a contribution due to the grain size.

An estimate of the number of detected diffracting grains $N_{D G}$ can be obtained from the average of the integrated intensities $I$ and their standard deviation $u(I)$ using Eq. 8.

It is recommended that the upper bound of the value of $u\left(2 \theta_{\text {grain }}\right)$ should be no more than $\approx \pm 0.015^{\circ}$. From Eq. 10 it can be seen that for a FWHM of $0.6^{\circ}$ the number of detected diffracting grains $N_{D G} \approx 72$. For a FWHM $=0.5^{\circ}, 0.4^{\circ}$ and $0.3^{\circ}$, then $N_{D G} \approx 50$, 32 and 18 respectively, The smaller the FWHM of the peak, the smaller the value of $N_{D G}$ needed. As a rule of thumb, the final stress uncertainties of greater than $40 \mathrm{MPa}$ in steel are too high and efforts to reduce the uncertainty should be implemented.

The time of measurement should not be too long otherwise the fitting uncertainty will be an underestimate of the total uncertainty. In order to increase the value of $N_{D G}$, oscillation of the specimen is recommended. If the value of $N_{D G}$ needed cannot be achieved then the method described by Holden et. al. [28] is recommended (if it is possible), where the specimen is measured and then rotated 180 degrees and measurement at the same place again and the result is averaged.

It has been shown that in some cases even the fitting uncertainty obtained from the peak fitting routine can be erroneous if the shape function is not appropriate for fitting the Bragg peak (assuming the detector has a linear response and is properly calibrated). This can be easily checked by measuring a reference specimen several times, with equal acquisition times, without moving it. The standard deviation of the $2 \theta_{0}$ values should be approximately equal to the average of the fitting uncertainties. Another good way to check if the fitting uncertainty is reasonable is by using Eq. 4 from Withers et. al. [8]. This will give the expected fitting uncertainty.

Knowing the size of grains and thus knowing the number of diffracting grains within a gauge volume can already give an indication as to whether one can rely on the commonly used standard uncertainty propagation or not. This can be worked out from the model presented. The results show that the model describes well situations where a specimen is not oscillated or oscillated around the omega $\omega$ axis during data acquisition. The largest oscillation used in this exercise was $+/-5^{\circ}$ in omega ( $\omega$-axis). The oscillation however should not be too large as to compromise the strain value measured in a particular direction.

The TG4 specimen has presented itself as an ideal specimen to highlight the problems associated with grain size. In the examples used in this case the parent region gives the least problems with grain size issues than the weld region where the grain size is much larger. For a grain size of around $70-100 \mu \mathrm{m}$ in the parent region, the fitting uncertainty seems to be adequate for the neutron measurements with the parameters used (if the sample is oscillated), which means the uncertainty contribution due to fitting is dominating the total uncertainty. 
For the weld region - with typical grain sizes of $140-260 \mu \mathrm{m}$ or more - the fitting uncertainty is certainly not adequate. Here caution should be taken to optimise the measurement by making sure the uncertainty due to grain size does not dominate the total uncertainty.

The four sets of residual stress data for the D-lines have been a good basis to apply the model. Special care was taken to compare data from the same specimen (3-1A) in order to eliminate any differences that may occur when measuring one of the other nominally identical specimens from the TG4 round robin. Indeed compared to the calculated robust average, the stresses in the weld region appear to be slightly less in the $(3-1 A)$. The robust average consisted of measurements on other nominally identical $(1-1 A)$ and $(2-1 A)$ specimens as well as the (3-1A).

One set of neutron data of the $D$ lines used a radial oscillating collimator for the secondary collimation of the neutrons. The stress results from that measurement agreed well with the other two sets of neutron data which utilized a slit for the secondary collimation. When using slits however, the secondary slit should not be more than 60 $\mathrm{mm}$ away from the centre of the gauge volume as standard good practice [27]. A slit suffers from aberrations similar to the pin-hole camera effect if the distances are larger. $A$ radial oscillating collimator eliminates this effect and can be used at a larger distance away from the centre of the gauge volume. The use of a secondary slit can also alter the shape of the peak, especially if one measures a relatively broad peak with a small slit width. The detected tails of the peak can be attenuated causing the peak to be less Gaussian in shape and thus the fitting program over estimates the uncertainty.

The next step should be the study the influence of texture. The concept of graininess and texture can become intertwined, as the presence of texture can either increase or decrease the number of grains seen on the detector. As can be seen from the coupons of TG4 SET Y, there appears to be texture, where the multiplicity ratio $\mathrm{m}^{311} / \mathrm{m}^{222}$ is not equal to 3 .

\section{Acknowledgements}

The allocation of beamtime by the ESRF and the support of the ID15 beamline staff is acknowledged. The fourth task group (TG4) of NeT is also gratefully acknowledged for making this work possible. 


\section{References}

[1] Smith, M.C.; Smith, A.C.; Wimpory, R.C. \& Ohms, C., "A review of the NeT Task Group 1 residual stress measurement and analysis round robin on a single weld bead-on-plate specimen" (2014). International Journal of Pressure Vessels and Piping 120-121, 93-140, doi: 10.1016/j.ijpvp.2014.05.002

[2] Wimpory, R. C., Ohms, C., Hofmann, M., Schneider, R. \& Youtsos, A. G. (2009). Int. J. Pres. Ves. Pip. 86, 48-62.

[3] Wimpory, R. C., Wasmuth, U., Rebelo-Kornmeier, J. \& Hofmann, M. (2010). Materials Science Forum, Vol. 638-642. Thermec 2009, Pts 1-4, edited by T. Chandra, N. Wanderka, W. Reimers \& M. Ionescu, pp. 2405-2410. Stafa-Zurich: Trans Tech Publications Ltd.

[4] DD CEN ISO/TS 21432:2005. Non-destructive testing. Standard test method for determining of residual stresses by neutron diffraction.

[5] Allen, A. J., Hutchings, M. T., Windsor, C. G. \& Andreani, C. (1985). Adv. Phys. 34, 445-473.

[6] Stacey, A., Macgillivary, H. J., Webster, G. A., Webster, P. J. \& Ziebeck, K. R. A. (1985). J. Strain Anal. Eng. Des. 20, 93-100.

[7] Allen, A., Andreani, C., Hutchings, M. T. \& Windsor, C. G. (1981). Ndt International 14, 249-254.

[8] Withers, P. J., Daymond, M. R. \& Johnson, M. W. (2001). J. Appl. Crystallogr. 34, 737-743.

[9] Smith, M. C. \& Smith, A. C. (2009). Int. J. Pres. Ves. Pip. 86, 79-95. 
[10] Wimpory, R. C., Mikula, P., Šaroun, J., Poeste, T., Li, J., Hofmann, M. \& Schneider, R. (2008). Neutron News 19, 16 - 19.

[11] Martins, R. V., Ohms, C. \& Decroos, K. (2010). Materials Science and Engineering: A 527, 4779-4787.

[12] R.V. Martins: NeT-Task Group 4: Three-Pass Slot Weld Specimen in Austenitic Stainless Steel, Protocol for the Destructive and Non-Destructive Determination of Residual Stress in Three-Pass Slot Weld Specimens in Austenitic Stainless Steel, Version 3.3, 2009, NeT document.

[13] Hofmann, M., Seidl, G. A., Rebelo-Kornmeier, J., Garbe, U., Schneider, R., Wimpory, R. C., Wasmuth, U. \& Noster, U. (2006). Materials Science Forum, Vol. 524-525. Residual Stresses VII, edited by W. Reimers \& S. Quander, pp. 211-216. Zurich-Uetikon: Trans Tech Publications Ltd.

[14] Ohms, C., Wimpory, R. C., Katsareas, D. E. \& Youtsos, A. G. (2009). Int. J. Pres. Ves. Pip. 86, 63-72.

[15] Ledbetter, H. M. (1981). Brit. J. Nondestr. Test. 286-287.

[16] Kröner, E. (1958). Z. Phys. 151, 504-518.

[17] Boin, M., Wimpory, R.C. \& Randau, C. (2014). Mater. Sci. Forum 777, 7-11, doi: 10.4028/www.scientific.net/MSF.777.7

[18] Wimpory, R.C. \& Ohms, C. (2010). Mater. Sci. Forum 638-642, 2487-2492, doi: 10.4028/www.scientific.net/MSF.638-642.2487.

[19] T. Gnaeupel-Herold, H. J. Prask, R. J. Fields, T. J. Foecke, Z. C. Xia, U. Lienert, A synchrotron study of residual stresses in a Al6022 deep drawn cup, Mater. Sci. Eng., A 366 (2004) 104-113. 
[20] C. Randau, U. Garbe, H.-G. Brokmeier, StressTextureCalculator : a software tool to extract texture, strain and microstructure information from area-detector measurements, J. Appl. Cryst. 44 (2011) 641-646.

[21] Martins, R. V. \& Honkimäki, V. (2003). Textures and Microstructures 35, 145 152.

[22] He, B. B. (2009). Two-Dimensional X-Ray Diffraction. John Wiley \& Sons, Inc.

[23] W.H. Press, e. a. (1992). Numerical Recipes. Cambridge University Press.

[24] Moysan, J., Apfel, A., Corneloup, G. \& Chassignole, B. (2003). Int. J. Pres. Ves. Pip. 80, 77-85.

[25] Pirling, T. (2000). Mater. Sci. Forum, Vol. 347-3. ECRS 5: Proceedings of the Fifth European Conference on Residual Stresses, edited by A. J. Bottger, R. Delhez \& E. J. Mittemeijer, pp. 107-112. Zurich-Uetikon: Trans Tech Publications Ltd.

[26] Hoffmann, J.-U. \& Schneider, R. (2004). TvTueb. Universität Tübingen, Germany.

[27] Pirling, T., Hughes, D. \& Robinson, J. S. (2010). Mater. Sci. Forum, Vol. 652. Mechanical Stress Evaluation by Neutrons and Synchrotron Radiation, edited by Y. Akiniwa, K. Akita \& H. Suzuki, pp. 80-85. Stafa-Zurich: Trans Tech Publications Ltd.

[28] Holden, T. M., Traore, Y., James, J., Kelleher, J., \& Bouchard, P. J. (2015). Determination and mitigation of the uncertainty of neutron diffraction measurements of residual strain in large-grained polycrystalline material. Journal of applied crystallography, 48(2), 0-0.

[29] R. C. Wimpory, Michael Hofmann, Joana Rebelo-Kornmeier, Mirko Boin and Carsten Ohms, "Minimizing and Characterizing Uncertainties in Neutron Strain Measurements with Special Attention to Grain Size Effects", Mater. Sci. Forum, Vol. 905, pp. 143-150, 2017 
Highlights

By presenting sets of diffraction data, obtained for an identical weld specimen on different strain scanning instruments, it has been demonstrated how grain size can influence the accuracy of a measurement. A simple model to estimate the extra random uncertainty contribution due to the so-called grain size statistics is applied and verified.

It has been shown that in some cases even the fitting uncertainty obtained from the peak fitting routine can be erroneous if the shape function is not appropriate for fitting the Bragg peak (assuming the detector has a linear response and is properly calibrated).

An estimate of the number of detected diffracting grains $N_{D G}$ needed for reasonable accuracies to be obtained can be garnered from a reference sample and the whole measurement can be designed based on this. The reference specimen can be used to optimise the time of an experiment, saving neutron beam time.

The benefit of continuous or stepwise oscillation to increase the number of detected grains on the detector is discussed. From the data obtained, best practice guidelines have been suggested on dealing with large grains when determining strain and stress with neutron diffraction. 